\title{
Asymptotic Analysis of General Multiuser Detectors in MIMO DS-CDMA Channels
}

\author{
Keigo Takeuchi, Toshiyuki Tanaka, Member, IEEE and Toru Yano
}

\begin{abstract}
We analyze a MIMO DS-CDMA channel with a general multiuser detector including a nonlinear multiuser detector, using the replica method. In the many-user, limit the MIMO DS-CDMA channel with the multiuser detector is decoupled into a bank of single-user SIMO Gaussian channels if a spatial spreading scheme is employed. On the other hand, it is decoupled into a bank of single-user MIMO Gaussian channels if a spatial spreading scheme is not employed. The spectral efficiency of the MIMO DS-CDMA channel with the spatial spreading scheme is comparable with that of the MIMO DS-CDMA channel using an optimal space-time block code without the spatial spreading scheme. In the case of the QPSK data modulation scheme the spectral efficiency of the MIMO DS-CDMA channel with the MMSE detector shows waterfall behavior and is very close to the corresponding sum capacity when the system load is just below the transition point of the waterfall behavior. Our result implies that the performance of a multiuser detector taking the data modulation scheme into consideration can be far superior to that of linear multiuser detectors.
\end{abstract}

\section{Index Terms}

Multiple-input multiple-output (MIMO) systems, direct-sequence code-division multiple access (DSCDMA), asymptotic analysis, multiuser detection, replica method.

K. Takeuchi is with the Department of Systems Science, Graduate School of Informatics, Kyoto University, Kyoto, 606-8501 Japan (e-mail: takeuchi@sys.i.kyoto-u.ac.jp).

T. Tanaka is with the Department of Systems Science, Graduate School of Informatics, Kyoto University, Kyoto, 606-8501 Japan (e-mail: tt@i.kyoto-u.ac.jp).

T. Yano is with the Department of Applied Physics and Physico-Informatics, Faculty of Science and Technology, Keio University, Kanagawa, 223-8522, Japan (e-mail: yano@thx.appi.keio.ac.jp). 


\section{INTRODUCTION}

Future wireless communications will utilize frequency multiplexing and spatial multiplexing to realize ultra high speed data communications [1]. Joint maximum likelihood (ML) decoding with high complexity is required to mitigate multiple access interference and attain the greatest possible frequency and spatial multiplexing gains. On the other hand, an affordable receiver structure with lower complexity is desired practically.

The key strategy to circumvent the complexity issue is to reduce a high-dimensional system to some lower-dimensional systems by making use of orthogonality. Strict orthogonality is utilized in Multicarrier (MC) schemes [1]-[3], orthogonal space-time block codes [4], and frequencydivision multiple access (FDMA). Although the strict orthogonality reduces the complexity, it can degrade the performance, as it is known [5] that FDMA is inferior to code-division multiple access (CDMA). A better trade-off between complexity and performance is provided by adopting statistical orthogonality. The direct-sequence CDMA (DS-CDMA) scheme, which is mathematically equivalent to multiple-input multiple-output (MIMO) systems with the Vertical Bell Labs Layered Space-Time (V-BLAST) architecture, decomposes joint decoding into multiuser detection and single-user decoding approximately, using the statistical orthogonality of spreading sequences. Although it has been reported in [6] that information loss due to spreading is negligible in a highly loaded system, there exists a large gap between the spectral efficiency of a DS-CDMA channel with a conventional linear multiuser detector front end [7] and the corresponding sum capacity [6]. The gap can be closed by nonlinear multiuser detection. The result in [8] implies that an optimal nonlinear multiuser detector has the capability of achieving very close performance to the corresponding sum capacity. An asymptotically optimal polynomial-time iterative detection algorithm on the basis of message passing has been proposed in [10]. Furthermore, Guo et al. [9] discovered the decoupling structure of the DS-CDMA channel under a general condition, due to the statistical orthogonality. An interesting observation is that the algorithm of [10] has the same decoupling structure as that of the optimal multiuser detector [11].

Recently, MIMO DS-CDMA systems, which are the application of MIMO technology to the DS-CDMA scheme, are being considered in anticipation of application to future wireless communications [12]-[15]. However, analysis of MIMO DS-CDMA systems is far from sufficient since many studies on MIMO DS-CDMA systems focus on conventional linear multiuser detectors 
[12], [14], [15], and it remains unclear how statistical orthogonality provided by both MIMO and DS-CDMA decomposes the MIMO DS-CDMA systems. Elucidation of the performance and structure of MIMO DS-CDMA systems with nonlinear multiuser detectors will help reveal the potential of MIMO DS-CDMA systems, and construct an algorithm of nonlinear multiuser detection. We thus perform an information-theoretical analysis of MIMO DS-CDMA systems under a general condition, including nonlinear multiuser detections.

The present paper is organized as follows. A MIMO DS-CDMA system model in a flat fading channel is introduced in Section 【II. Our main results are summarized in Section III and the numerical evaluations are described in Section IV]. We discuss nonlinear multiuser detection and the extension of the results in Section V. Section VI presents our conclusions.

Throughout the present paper, $\boldsymbol{A}^{T}$ and $\boldsymbol{A}^{H}$ denote the transpose of $\boldsymbol{A}$ and the conjugate transpose of $\boldsymbol{A}$, respectively. $\mathcal{C N}(\mathbf{0}, \boldsymbol{\Sigma})$ stands for the circularly symmetric zero-mean complex Gaussian distribution with the covariance matrix $\Sigma$. $\boldsymbol{e}_{N}$ represents the $N$-dimensional vector of which the elements are all one. $p\left(\boldsymbol{y} \mid \boldsymbol{x},\left\{\boldsymbol{A}_{j}\right\} ; \boldsymbol{\Theta}\right)$ denotes the probability density function of $\boldsymbol{y}$ conditioned on $\boldsymbol{x}$ and $\left\{\boldsymbol{A}_{j}\right\}$ with the parameter $\boldsymbol{\Theta} . \log a$ and $\ln a$ stand for $\log _{2} a$ and $\log _{\mathrm{e}} a$, respectively. $\mathrm{KL}(\cdot \| \cdot)$ represents the Kullback-Leibler divergence with the logarithm to base 2. $|\mathcal{A}|, \otimes, \operatorname{Re}(a)$, and $\operatorname{Im}(a)$ denotes the number of elements of a set $\mathcal{A}$, the Kronecker product operator between two matrices, the real and imaginary parts of a complex number $a$, respectively.

\section{MODEL}

\section{A. MIMO DS-CDMA Channel}

We consider an uplink of a chip-synchronous MIMO DS-CDMA channel with $K$ users [12]. The $k$ th user and the receiver have $M_{k}$ transmit antennas and $N$ receive antennas, respectively. A space-time encoder for the $k$ th user maps its message into a codeword matrix, each element of which is mapped into a symbol by a data modulator. The product of each symbol $x_{m}^{k}$, the variance of which is $P_{m}^{k}$ (received power), and a random spreading sequence $\left\{s_{l m}^{k} ; l=1, \ldots, L\right\}$ with the spreading factor $L$ is then transmitted from the $m$ th transmit antenna of the $k$ th user. The $N$-dimensional received signal vector $\boldsymbol{y}_{l}$ propagated through a flat fading channel is given by

$$
\boldsymbol{y}_{l}=\sum_{k=1}^{K} \boldsymbol{H}^{k} \boldsymbol{S}_{l}^{k} \boldsymbol{x}^{k}+\boldsymbol{n}_{l}, \quad l=1, \ldots, L
$$


where $\boldsymbol{x}^{k}=\left(x_{1}^{k}, \ldots, x_{M_{k}}^{k}\right)^{T}$ is the symbol vector for the $k$ th user; the $M_{k} \times M_{k}$ diagonal matrix $\boldsymbol{S}_{l}^{k}$ is given by $\boldsymbol{S}_{l}^{k}=\operatorname{diag}\left(s_{l 1}^{k}, \ldots, s_{l M_{k}}^{k}\right) ; \boldsymbol{H}^{k}=\left(\boldsymbol{h}_{1}^{k}, \ldots, \boldsymbol{h}_{M_{k}}^{k}\right)$ is the $N \times M_{k}$ random channel matrix for the $k$ th user, i.e., the $(n, m)$-element $h_{n m}^{k}$ of $\boldsymbol{H}^{k}$ represents the complex channel gain from the $m_{k}$ th transmit antenna of the $k$ th user to the $n$th receive antenna; and $\boldsymbol{n}_{l} \sim$ $\mathcal{C N}\left(\mathbf{0}, N_{0} \boldsymbol{I}_{N}\right)$ is additive white Gaussian noise (AWGN). The MIMO DS-CDMA channel (1) can be expressed as the vector channel of higher dimension,

$$
\vec{y}=\mathcal{A} \vec{x}+\vec{n}
$$

where $\overrightarrow{\boldsymbol{x}}=\left(\boldsymbol{x}^{1^{T}}, \ldots, \boldsymbol{x}^{K^{T}}\right)^{T}$ is the entire symbol vector; $\overrightarrow{\boldsymbol{y}}=\left(\boldsymbol{y}_{1}{ }^{T}, \ldots, \boldsymbol{y}_{L}{ }^{T}\right)^{T}$ represents the entire received signal vector in a symbol period; $\overrightarrow{\boldsymbol{n}}=\left(\boldsymbol{n}_{1}{ }^{T}, \ldots, \boldsymbol{n}_{L}{ }^{T}\right)^{T}$ is the entire noise vector; and the matrix $\mathcal{A}$ is given by

$$
\mathcal{A}=\left[\begin{array}{ccc}
\boldsymbol{H}^{1} \boldsymbol{S}_{1}^{1} & \cdots & \boldsymbol{H}^{K} \boldsymbol{S}_{1}^{K} \\
\vdots & & \vdots \\
\boldsymbol{H}^{1} \boldsymbol{S}_{L}^{1} & \cdots & \boldsymbol{H}^{K} \boldsymbol{S}_{L}^{K}
\end{array}\right] .
$$

We consider a space-time spreading (STS) scheme, which is implemented with independent spreading sequences from antenna to antenna (Fig. 1), and a time spreading (TS) scheme, which uses the same spreading sequences for different antennas of each user (Fig. 22).

Assumption 1: The real part and the imaginary part of $\left\{\left(s_{l 1}^{k}, \ldots, s_{l M_{k}}^{k}\right)^{T} ; k \in \mathcal{K}=\{1, \ldots, K\}\right.$, $l=1, \ldots, L\}$ are independent and identically distributed (i.i.d.) zero-mean random vectors with the covariance matrix $\left[\delta \boldsymbol{e}_{M_{k}} \boldsymbol{e}_{M_{k}}^{T}+(1-\delta) \boldsymbol{I}_{M_{k}}\right] /(2 L)$.

Letting $\delta=0$ and 1 in Assumption 1 correspond to the STS and TS schemes, respectively. We further assume that the users are divided into $P$ groups, in each of which the users employ the same number of transmit antennas and the same data modulation scheme.

Assumption 2: For a partition $\left\{\mathcal{K}_{p} ; p=1, \ldots, P\right\}$ of $\mathcal{K},\left\{\boldsymbol{x}^{k} ; k \in \mathcal{K}_{p}\right\}$ are i.i.d. complex random vectors, and the symbol vectors of users who belong to different groups are mutually independent.

Assumption 3: The moment generating function of $x_{m}^{k}$ exists in the neighborhood of the origin.

We remark that Assumption 2 includes the case in which the number of the groups is equal to the number of users and that Assumption 3 holds in the case of the Gaussian data modulation as well as any conventional digital data modulations. Although we need these assumptions in 
order to derive part of results described in the next Section, we believe that Assumption 2 can be relaxed.

\section{B. Receiver Structure}

Assuming the channel side information (CSI) only at the receiver, we consider separate decoding, where a multiuser detector front end feeds soft or hard decisions to single-user spacetime decoders. The multiuser detector postulates that the prior distribution of the symbol vector for the $k$ th user is $p\left(\tilde{\boldsymbol{x}}^{k}\right)$, the moment generating function of which is assumed to exist in the neighborhood of the origin, and that the variance of the noise is $\tilde{N}_{0}$. Tildes indicate that the postulated prior distribution and the postulated variance of the noise need not coincide with the actual ones. The multiuser detector estimates the symbol vector of each user on the basis of the generalized posterior mean estimator (GPME) [9]

$$
\left\langle\tilde{\boldsymbol{x}}^{k}\right\rangle=\frac{\mathrm{E}_{\overrightarrow{\tilde{\boldsymbol{x}}}}\left[\tilde{\boldsymbol{x}}^{k} p\left(\overrightarrow{\overrightarrow{\boldsymbol{y}}}=\overrightarrow{\boldsymbol{y}} \mid \overrightarrow{\overrightarrow{\boldsymbol{x}}}, \mathcal{A} ; \tilde{N}_{0}\right)\right]}{\mathrm{E}_{\overrightarrow{\tilde{\boldsymbol{x}}}}\left[p\left(\overrightarrow{\tilde{\boldsymbol{y}}}=\overrightarrow{\boldsymbol{y}} \mid \overrightarrow{\tilde{\boldsymbol{x}}}, \mathcal{A} ; \tilde{N}_{0}\right)\right]},
$$

where $\overrightarrow{\tilde{\boldsymbol{x}}}=\left(\left(\tilde{\boldsymbol{x}}^{1}\right)^{T}, \ldots,\left(\tilde{\boldsymbol{x}}^{K}\right)^{T}\right)^{T}$ and $p\left(\overrightarrow{\tilde{\boldsymbol{y}}} \mid \overrightarrow{\tilde{\boldsymbol{x}}}, \mathcal{A} ; \tilde{N}_{0}\right)$ represents the channel postulated by the receiver:

$$
\overrightarrow{\tilde{\boldsymbol{y}}}=\mathcal{A} \overrightarrow{\tilde{\boldsymbol{x}}}+\overrightarrow{\tilde{\boldsymbol{n}}}, \quad \overrightarrow{\tilde{\boldsymbol{n}}} \sim \mathcal{C N}\left(\mathbf{0}, \tilde{N}_{0} \boldsymbol{I}_{N L}\right)
$$

The multiuser detector (4) corresponds to the minimum mean-squared error (MMSE) detector if $p\left(\tilde{\boldsymbol{x}}^{k}\right)$ and $\tilde{N}_{0}$ coincide with $p\left(\boldsymbol{x}^{k}\right)$ and $N_{0}$. The multiuser detector becomes a linear detector if $\tilde{\boldsymbol{x}}^{k}$ follows a zero-mean circularly symmetric complex Gaussian distribution. Furthermore, the linear detector corresponds to the linear MMSE detector if $\tilde{N}_{0}$ coincides with $N_{0}$.

The maximum spectral efficiency 1 per chip of the MIMO DS-CDMA channel with CSI at the receiver is given by the mutual information between $\overrightarrow{\boldsymbol{x}}$ and $\overrightarrow{\boldsymbol{y}}$ [16]

$$
\mathcal{C}_{\text {joint }}=\frac{1}{L} I(\overrightarrow{\boldsymbol{x}} ; \overrightarrow{\boldsymbol{y}})=\frac{1}{L} \mathrm{E}\left[\log \frac{p\left(\overrightarrow{\boldsymbol{y}} \mid \overrightarrow{\boldsymbol{x}}, \mathcal{A} ; N_{0}\right)}{\mathrm{E}_{\overrightarrow{\boldsymbol{x}}}\left[p\left(\overrightarrow{\boldsymbol{y}} \mid \overrightarrow{\boldsymbol{x}}, \mathcal{A} ; N_{0}\right)\right]} \mid \mathcal{A}\right] .
$$

On the other hand, the spectral efficiency per chip of the MIMO DS-CDMA channel with the GPME detector front end is given by

$$
\mathcal{C}_{\text {sep }}=\frac{1}{L} \sum_{k=1}^{K} I\left(\boldsymbol{x}^{k} ;\left\langle\tilde{\boldsymbol{x}}^{k}\right\rangle\right)=\frac{1}{L} \sum_{k=1}^{K} \mathrm{E}\left[\log \frac{p\left(\left\langle\tilde{\boldsymbol{x}}^{k}\right\rangle \mid \boldsymbol{x}^{k}, \mathcal{A}\right)}{\mathrm{E}_{\boldsymbol{x}^{k}}\left[p\left(\left\langle\tilde{\boldsymbol{x}}^{k}\right\rangle \mid \boldsymbol{x}^{k}, \mathcal{A}\right)\right]} \mid \mathcal{A}\right] .
$$

\footnotetext{
${ }^{1}$ Note that in the present paper the term "maximum spectral efficiency" refers to the spectral efficiency of a channel with an optimal receiver structure on condition that a data modulation scheme and power allocation are specified.
} 


\section{MAIn Results}

The main results comprise analytical formulas for $\mathcal{C}_{\text {joint }}$ (Proposition 1) and $\mathcal{C}_{\text {sep }}$ (Proposition (3), and decoupling results (Proposition 2). First, we list some definitions.

Definition 1: A single-input multiple-output (SIMO) Gaussian channel for the $m$ th transmit antenna of the $k$ th user, a postulated SIMO Gaussian channel for the $m$ th transmit antenna of the $k$ th user, a MIMO Gaussian channel for the $k$ th user, and a postulated MIMO Gaussian channel for the $k$ th user are respectively defined as

$$
\begin{aligned}
\boldsymbol{y}_{m}^{k}=\boldsymbol{h}_{m}^{k} x_{m}^{k}+\boldsymbol{n}_{m}^{k}, & \boldsymbol{n}_{m}^{k} \sim \mathcal{C N}(\mathbf{0}, \boldsymbol{R}), \\
\tilde{\boldsymbol{y}}_{m}^{k}=\boldsymbol{h}_{m}^{k} \tilde{x}_{m}^{k}+\tilde{\boldsymbol{n}}_{m}^{k}, & \tilde{\boldsymbol{n}}_{m}^{k} \sim \mathcal{C N}(\mathbf{0}, \tilde{\boldsymbol{R}}), \\
\boldsymbol{y}^{k}=\boldsymbol{H}^{k} \boldsymbol{x}^{k}+\boldsymbol{n}^{k}, & \boldsymbol{n}^{k} \sim \mathcal{C N}(\mathbf{0}, \boldsymbol{W}), \\
\tilde{\boldsymbol{y}}^{k}=\boldsymbol{H}^{k} \tilde{\boldsymbol{x}}^{k}+\tilde{\boldsymbol{n}}^{k}, & \tilde{\boldsymbol{n}}^{k} \sim \mathcal{C \mathcal { N }}(\mathbf{0}, \tilde{\boldsymbol{W}}),
\end{aligned}
$$

where $\boldsymbol{R}, \tilde{\boldsymbol{R}}, \boldsymbol{W}$, and $\tilde{\boldsymbol{W}}$ are $N \times N$ positive definite Hermitian matrices.

Definition 2: The GPME of $x_{m}^{k}$ in the SIMO Gaussian channel (8a) and the GPME of $\boldsymbol{x}^{k}$ in the MIMO Gaussian channel (8c) are respectively given by

$$
\begin{aligned}
\left\langle\tilde{x}_{m}^{k}\right\rangle_{\text {SIMO }} & =\frac{\mathrm{E}_{\tilde{x}_{m}^{k}}\left[\tilde{x}_{m}^{k} p\left(\tilde{\boldsymbol{y}}_{m}^{k}=\boldsymbol{y}_{m}^{k} \mid \tilde{x}_{m}^{k}, \boldsymbol{h}_{m}^{k} ; \tilde{\boldsymbol{R}}\right)\right]}{\mathrm{E}_{\tilde{x}_{m}^{k}}\left[p\left(\tilde{\boldsymbol{y}}_{m}^{k}=\boldsymbol{y}_{m}^{k} \mid \tilde{x}_{m}^{k}, \boldsymbol{h}_{m}^{k} ; \tilde{\boldsymbol{R}}\right)\right]}, \\
\left\langle\tilde{\boldsymbol{x}}^{k}\right\rangle_{\mathrm{MIMO}} & =\frac{\mathrm{E}_{\tilde{\boldsymbol{x}}^{k}}\left[\tilde{\boldsymbol{x}}^{k} p\left(\tilde{\boldsymbol{y}}^{k}=\boldsymbol{y}^{k} \mid \tilde{\boldsymbol{x}}^{k}, \boldsymbol{H}^{k} ; \tilde{\boldsymbol{W}}\right)\right]}{\mathrm{E}_{\tilde{\boldsymbol{x}}^{k}}\left[p\left(\tilde{\boldsymbol{y}}^{k}=\boldsymbol{y}^{k} \mid \tilde{\boldsymbol{x}}^{k}, \boldsymbol{H}^{k} ; \tilde{\boldsymbol{W}}\right)\right]} .
\end{aligned}
$$

Definition 3: The maximum spectral efficiencies of the SIMO Gaussian channel (8a) and the MIMO Gaussian channel (8c) are respectively given by

$$
\begin{gathered}
\mathcal{C}_{\mathrm{SIMO}}^{k, m}(\boldsymbol{R})=\mathrm{E}\left\{\log \frac{p\left(\boldsymbol{y}_{m}^{k} \mid x_{m}^{k}, \boldsymbol{h}_{m}^{k} ; \boldsymbol{R}\right)}{\mathrm{E}_{x_{m}^{k}}\left[p\left(\boldsymbol{y}_{m}^{k} \mid x_{m}^{k}, \boldsymbol{h}_{m}^{k} ; \boldsymbol{R}\right)\right]} \mid \boldsymbol{h}_{m}^{k}\right\}, \\
\mathcal{C}_{\mathrm{MIMO}}^{k}(\boldsymbol{W})=\mathrm{E}\left\{\log \frac{p\left(\boldsymbol{y}^{k} \mid \boldsymbol{x}^{k}, \boldsymbol{H}^{k} ; \boldsymbol{W}\right)}{\mathrm{E}_{\boldsymbol{x}^{k}}\left[p\left(\boldsymbol{y}^{k} \mid \boldsymbol{x}^{k}, \boldsymbol{H}^{k} ; \boldsymbol{W}\right)\right]} \mid \boldsymbol{H}^{k}\right\} .
\end{gathered}
$$

Definition 4: The mean-squared error of the GPME (9a), the generalized posterior variance of the SIMO Gaussian channel (8a), the error covariance matrix of the GPME (9b), and the generalized posterior covariance matrix of the MIMO Gaussian channel $8 \mathrm{CC}$ are respectively 
defined as

$$
\begin{aligned}
\mathcal{E}_{\mathrm{SIMO}}^{k, m}(\boldsymbol{R}, \tilde{\boldsymbol{R}}) & =\mathrm{E}\left[\left|x_{m}^{k}-\left\langle\tilde{x}_{m}^{k}\right\rangle_{\mathrm{SIMO}}\right|^{2} \mid \boldsymbol{h}_{m}^{k}\right], \\
\mathcal{V}_{\mathrm{SIMO}}^{k, m}(\boldsymbol{R}, \tilde{\boldsymbol{R}}) & =\mathrm{E}\left[\left|\tilde{x}_{m}^{k}-\left\langle\tilde{x}_{m}^{k}\right\rangle_{\mathrm{SIMO}}\right|^{2} \mid \boldsymbol{h}_{m}^{k}\right], \\
\mathcal{E}_{\mathrm{MIMO}}^{k}(\boldsymbol{W}, \tilde{\boldsymbol{W}}) & =\mathrm{E}\left[\left(\boldsymbol{x}^{k}-\left\langle\tilde{\boldsymbol{x}}^{k}\right\rangle_{\mathrm{MIMO}}\right)\left(\boldsymbol{x}^{k}-\left\langle\tilde{\boldsymbol{x}}^{k}\right\rangle_{\mathrm{MIMO}}\right)^{H} \mid \boldsymbol{H}^{k}\right], \\
\mathcal{V}_{\mathrm{MIMO}}^{k}(\boldsymbol{W}, \tilde{\boldsymbol{W}}) & =\mathrm{E}\left[\left(\tilde{\boldsymbol{x}}^{k}-\left\langle\tilde{\boldsymbol{x}}^{k}\right\rangle_{\mathrm{MIMO}}\right)\left(\tilde{\boldsymbol{x}}^{k}-\left\langle\tilde{\boldsymbol{x}}^{k}\right\rangle_{\mathrm{MIMO}}\right)^{H} \mid \boldsymbol{H}^{k}\right] .
\end{aligned}
$$

In order to evaluate the maximum spectral efficiency (6) analytically, we consider the manyuser limit in which both the number of users $K$ and the spreading factor $L$ tend toward infinity with their ratio $\beta=K / L$ fixed, assuming the following:

Assumption 4: The spectral efficiencies (6) and (7) satisfy the self-averaging property with respect to $\mathcal{S}$, i.e., (6) and (7) converge in probability respectively to the expectations of (6) and (7) with respect to $\mathcal{S}=\left\{\boldsymbol{S}_{l}^{k} ; l=1, \ldots, L, k \in \mathcal{K}\right\}$ in the many-user limit.

The many-user limit has been considered in many previous studies [6]-[9], [12], [15], [17], [18]. The self-averaging property of the MIMO DS-CDMA channel has partially been proved under the assumption of the Gaussian data modulation, i.e., the self-averaging property of (7) has been proved for the STS scheme [12], [17], and has been proved for the TS scheme for the case of $N=1$ or $M_{k} \rightarrow \infty$ [12]. Since proving the self-averaging property for an arbitrary prior distribution of the symbol vector is a challenging problem, in this paper, as in previous studies [8], [9], the self-averaging property is assumed .

Proposition 1: For arbitrary realizations of $\mathcal{S}$ and $\mathcal{H}=\left\{\boldsymbol{H}^{k} ; k \in \mathcal{K}\right\}$, in the many-user limit the maximum spectral efficiencies (6) of the MIMO DS-CDMA channels with the STS and TS schemes are respectively given by

$$
\begin{aligned}
& \mathcal{C}_{\text {joint }}^{\mathrm{STS}}=\beta \lim _{K \rightarrow \infty} \frac{1}{K} \sum_{k=1}^{K} \sum_{m=1}^{M_{k}} \mathcal{C}_{\mathrm{SIMO}}^{k, m}(\boldsymbol{R})+\mathrm{KL}\left(\mathcal{C N}\left(\mathbf{0}, N_{0} \boldsymbol{I}_{N}\right) \| \mathcal{C N}(\mathbf{0}, \boldsymbol{R})\right) \quad \text { for STS } \\
& \mathcal{C}_{\text {joint }}^{\mathrm{TS}}=\beta \lim _{K \rightarrow \infty} \frac{1}{K} \sum_{k=1}^{K} \mathcal{C}_{\mathrm{MIMO}}^{k}(\boldsymbol{W})+\mathrm{KL}\left(\mathcal{C N}\left(\mathbf{0}, N_{0} \boldsymbol{I}_{N}\right) \| \mathcal{C N}(\mathbf{0}, \boldsymbol{W})\right) \quad \text { for } \mathrm{TS},
\end{aligned}
$$

where $\boldsymbol{R}$ is a solution of the fixed-point equation

$$
\boldsymbol{R}=N_{0} \boldsymbol{I}_{N}+\beta \lim _{K \rightarrow \infty} \frac{1}{K} \sum_{k=1}^{K} \sum_{m=1}^{M_{k}} \mathcal{E}_{\text {SIMO }}^{k, m}(\boldsymbol{R}, \boldsymbol{R}) \boldsymbol{h}_{m}^{k}\left(\boldsymbol{h}_{m}^{k}\right)^{H},
$$

and $\boldsymbol{W}$ satisfies the fixed-point equation

$$
\boldsymbol{W}=N_{0} \boldsymbol{I}_{N}+\beta \lim _{K \rightarrow \infty} \frac{1}{K} \sum_{k=1}^{K} \boldsymbol{H}^{k} \mathcal{E}_{\mathrm{MIMO}}^{k}(\boldsymbol{W}, \boldsymbol{W})\left(\boldsymbol{H}^{k}\right)^{H} .
$$


In the case in which there exist multiple solutions of $\boldsymbol{R}$ and $\boldsymbol{W}$, one should choose the solutions so as to minimize the maximum spectral efficiencies (12a) and (12b), respectively.

The derivation of Proposition 1 is summarized in Appendix $\mathrm{A}$. The operational meaning of the expressions (12a) and $(12 \mathrm{~b}$ ) is that the first terms in the right-hand sides are the spectral efficiencies of the MIMO DS-CDMA channel with the MMSE detector front end in the cases of the STS scheme and the TS scheme, respectively, and the second terms in the right-hand sides are the separation loss, i.e., the information loss due to the separation of detection and decoding. The following lemma is needed in order to derive the propositions providing this interpretation.

Lemma 1: If $\left\{\boldsymbol{H}^{k} ; k \in \mathcal{K}_{p}\right\}$ are i.i.d., in the limit $\left|\mathcal{K}_{1}\right| \rightarrow \infty, \ldots,\left|\mathcal{K}_{P}\right| \rightarrow \infty$, and $L \rightarrow \infty$ with their ratios $\left\{\beta_{p}=\left|\mathcal{K}_{p}\right| / L ; p=1, \ldots, P\right\}$ fixed, an arbitrary joint moment of the symbol vector and the GPME (4) for a user $k \in \mathcal{K}_{p}$ converges to the corresponding joint moment of the symbol vector and its GPME in a single-user channel, i.e.,

$$
\begin{aligned}
& \lim _{\left|\mathcal{K}_{1}\right|, \ldots,\left|\mathcal{K}_{P}\right|, L \rightarrow \infty} \mathrm{E}\left[\prod_{m=1}^{M_{k}}\left\{\left[\operatorname{Re}\left(x_{m}^{k}\right)\right]^{i_{m}^{(\mathrm{r})}}\left[\operatorname{Im}\left(x_{m}^{k}\right)\right]^{i_{m}^{(\mathrm{i})}}\left\langle\operatorname{Re}\left(\tilde{x}_{m}^{k}\right)\right\rangle^{j_{m}^{(\mathrm{r})}}\left\langle\operatorname{Im}\left(\tilde{x}_{m}^{k}\right)\right\rangle^{j_{m}^{(\mathrm{i})}}\right\}\right] \\
& = \begin{cases}\mathrm{E}\left[\prod_{m=1}^{M_{k}}\left\{\left[\operatorname{Re}\left(x_{m}^{k}\right)\right]^{\left.i_{m}^{\mathrm{r}}\right)}\left[\operatorname{Im}\left(x_{m}^{k}\right)\right]^{i_{m}^{(\mathrm{i})}}\left\langle\operatorname{Re}\left(\tilde{x}_{m}^{k}\right)\right\rangle_{\mathrm{SIMO}}^{j_{m}^{(\mathrm{r})}}\left\langle\operatorname{Im}\left(\tilde{x}_{m}^{k}\right)\right\rangle_{\mathrm{SIMO}}^{j_{m}^{(\mathrm{i})}}\right\}\right] & \text { for STS, } \\
\mathrm{E}\left[\prod_{m=1}^{M_{k}}\left\{\left[\operatorname{Re}\left(x_{m}^{k}\right)\right]^{i_{m}^{(\mathrm{r})}}\left[\operatorname{Im}\left(x_{m}^{k}\right)\right]^{i_{m}^{(\mathrm{i})}}\left\langle\operatorname{Re}\left(\tilde{x}_{m}^{k}\right)\right\rangle_{\mathrm{MIMO}}^{j_{m}^{(\mathrm{r})}}\left\langle\operatorname{Im}\left(\tilde{x}_{m}^{k}\right)\right\rangle_{\mathrm{MIMO}}^{j_{m}^{(\mathrm{i})}}\right\}\right] & \text { for TS, }\end{cases}
\end{aligned}
$$

and $(\boldsymbol{W}, \tilde{\boldsymbol{W}})$ is a solution of the following fixed-point equations:

$$
\begin{aligned}
& \boldsymbol{W}=N_{0} \boldsymbol{I}_{N}+\beta \lim _{K \rightarrow \infty} \frac{1}{K} \sum_{k=1}^{K} \boldsymbol{H}^{k} \mathcal{E}_{\mathrm{MIMO}}^{k}(\boldsymbol{W}, \tilde{\boldsymbol{W}})\left(\boldsymbol{H}^{k}\right)^{H} \\
& \tilde{\boldsymbol{W}}=\tilde{N}_{0} \boldsymbol{I}_{N}+\beta \lim _{K \rightarrow \infty} \frac{1}{K} \sum_{k=1}^{K} \boldsymbol{H}^{k} \mathcal{V}_{\mathrm{MIMO}}^{k}(\boldsymbol{W}, \tilde{\boldsymbol{W}})\left(\boldsymbol{H}^{k}\right)^{H}
\end{aligned}
$$


In the case in which there exist multiple solutions of $(\boldsymbol{R}, \tilde{\boldsymbol{R}})$ and $(\boldsymbol{W}, \tilde{\boldsymbol{W}})$, one should respectively choose the solutions that minimize the free energies

$$
\frac{\beta}{\ln 2} \mathcal{F}=\left\{\begin{array}{l}
\beta \lim _{K \rightarrow \infty} \frac{1}{K} \sum_{k=1}^{K} \sum_{m=1}^{M_{k}} \tilde{\mathcal{C}}_{\mathrm{SIMO}}^{k, m}(\boldsymbol{R}, \tilde{\boldsymbol{R}})+N \log \left(\pi \mathrm{e} N_{0}\right)+F(\boldsymbol{R}, \tilde{\boldsymbol{R}}) \text { for STS } \\
\beta \lim _{K \rightarrow \infty} \frac{1}{K} \sum_{k=1}^{K} \tilde{\mathcal{C}}_{\mathrm{MIMO}}^{k}(\boldsymbol{W}, \tilde{\boldsymbol{W}})+N \log \left(\pi \mathrm{e} N_{0}\right)+F(\boldsymbol{W}, \tilde{\boldsymbol{W}}) \text { for TS }
\end{array}\right.
$$

where $\tilde{\mathcal{C}}_{\text {SIMO }}^{k, m}(\boldsymbol{R}, \tilde{\boldsymbol{R}})$ and $\tilde{\mathcal{C}}_{\mathrm{MIMO}}^{k}(\boldsymbol{W}, \tilde{\boldsymbol{W}})$ are given by

$$
\begin{gathered}
\tilde{\mathcal{C}}_{\mathrm{SIMO}}^{k, m}(\boldsymbol{R}, \tilde{\boldsymbol{R}})=\int p\left(\boldsymbol{y}_{m}^{k}, x_{m}^{k} \mid \boldsymbol{h}_{m}^{k} ; \boldsymbol{R}\right) \log \frac{p\left(\boldsymbol{y}_{m}^{k} \mid x_{m}^{k}, \boldsymbol{h}_{m}^{k} ; \tilde{\boldsymbol{R}}\right)}{\mathrm{E}_{\tilde{x}_{m}^{k}}\left[p\left(\tilde{\boldsymbol{y}}_{m}^{k}=\boldsymbol{y}_{m}^{k} \mid \tilde{x}_{m}^{k}, \boldsymbol{h}_{m}^{k} ; \tilde{\boldsymbol{R}}\right)\right]} d x_{m}^{k} \boldsymbol{d} \boldsymbol{y}_{m}^{k}, \\
\tilde{\mathcal{C}}_{\mathrm{MIMO}}^{k}(\boldsymbol{W}, \tilde{\boldsymbol{W}})=\int p\left(\boldsymbol{y}^{k}, \boldsymbol{x}^{k} \mid \boldsymbol{H}^{k} ; \boldsymbol{W}\right) \log \frac{p\left(\boldsymbol{y}^{k} \mid \boldsymbol{x}^{k}, \boldsymbol{H}^{k} ; \tilde{\boldsymbol{W}}\right)}{\mathrm{E}_{\tilde{\boldsymbol{x}}^{k}}\left[p\left(\tilde{\boldsymbol{y}}^{k}=\boldsymbol{y}^{k} \mid \tilde{\boldsymbol{x}}^{k}, \boldsymbol{H}^{k} ; \tilde{\boldsymbol{W}}\right)\right]} \boldsymbol{d} \boldsymbol{x}^{k} \boldsymbol{d} \boldsymbol{y}^{k},
\end{gathered}
$$

and the function $F(\cdot, \tilde{*})$ is defined as

$$
\begin{aligned}
& F(\boldsymbol{A}, \tilde{\boldsymbol{A}})=\mathrm{KL}\left(\mathcal{C N}\left(\mathbf{0}, N_{0} \boldsymbol{I}_{N}\right) \| \mathcal{C N}(\mathbf{0}, \tilde{\boldsymbol{A}})\right)+\mathrm{KL}(\mathcal{C N}(\mathbf{0}, \boldsymbol{A}) \| \mathcal{C N}(\mathbf{0}, \tilde{\boldsymbol{A}})) \\
& +\mathrm{KL}\left(\mathcal{C N}\left(\mathbf{0}, \tilde{N}_{0} \boldsymbol{I}_{N}\right) \| \mathcal{C N}(\mathbf{0}, \tilde{\boldsymbol{A}})\right)-\mathrm{KL}\left(\mathcal{C N}\left(\mathbf{0}, \tilde{N}_{0} \boldsymbol{I}_{N}\right) \| \mathcal{C N}\left(\mathbf{0}, \tilde{\boldsymbol{A}} \boldsymbol{A}^{-1} \tilde{\boldsymbol{A}}\right)\right)
\end{aligned}
$$

Lemma 1 is derived in Appendix B. From Assumption 3, the moment generating functions of the GPMEs (9a) and (9b) are expected to exist in the neighborhood of the origin, respectively. Then, the joint moment sequences (15a) and (15b) uniquely determine the respective joint distributions. Furthermore, assuming that all joint moments of the symbol vector and the GPME (4) have the self-averaging property with respect to $\mathcal{S}$, we obtain the following decoupling result.

Proposition 2: If $\left\{x_{m}^{k}, \tilde{x}_{m}^{k} ; m=1, \ldots, M_{k}\right\}$ are mutually independent and $\left\{\boldsymbol{H}^{k} ; k \in \mathcal{K}_{p}\right\}$ are i.i.d., in the limit $\left|\mathcal{K}_{1}\right| \rightarrow \infty, \ldots,\left|\mathcal{K}_{P}\right| \rightarrow \infty$, and $L \rightarrow \infty$ with $\left\{\beta_{p}\right\}$ fixed, then the conditional joint distribution of $\boldsymbol{x}^{k}$ and $\left\langle\boldsymbol{x}^{k}\right\rangle$ conditioned on $\mathcal{S}$ and $\mathcal{H}$ converges to the joint distribution of $\boldsymbol{x}^{k}$ and its GPME in a single-user channel, i.e.,

$$
p\left(\boldsymbol{x}^{k},\left\langle\tilde{\boldsymbol{x}}^{k}\right\rangle \mid \mathcal{S}, \mathcal{H}\right) \rightarrow \begin{cases}\prod_{m=1}^{M_{k}} p\left(x_{m}^{k},\left\langle\tilde{x}_{m}^{k}\right\rangle_{\mathrm{SIMO}} \mid \boldsymbol{h}_{m}^{k}\right) & \text { for STS } \\ p\left(\boldsymbol{x}^{k},\left\langle\tilde{\boldsymbol{x}}^{k}\right\rangle_{\mathrm{MIMO}} \mid \boldsymbol{H}^{k}\right) & \text { for TS }\end{cases}
$$

with $(\boldsymbol{R}, \tilde{\boldsymbol{R}})$ and $(\boldsymbol{W}, \tilde{\boldsymbol{W}})$ defined in Lemma 1

Although in order to simplify the derivation of Proposition 2 , we have assumed that the symbol vectors and the channel matrices are i.i.d. in the same group $\mathcal{K}_{p}$, we conjecture that this assumption is not required. Proposition 2 implies that the decoupling principle [9] holds in the 
MIMO DS-CDMA channel, i.e., in the case of the STS scheme the MIMO DS-CDMA channel (1) with the GPME detector front end (4) is decoupled into a bank of the SIMO Gaussian channels (8a) with the GPME detector front ends (9a) in the many-user limit, and in the case of the TS scheme the MIMO DS-CDMA channel (1) with the GPME detector front end (4) is decoupled into a bank of the MIMO Gaussian channels $8 \mathrm{c}$ ) with the GPME detector front ends (9b) in the many-user limit. From this decoupling result we can evaluate the spectral efficiency (7) of the MIMO DS-CDMA channel (1) with the GPME detector front end (4).

Proposition 3: If $\left\{x_{m}^{k}, \tilde{x}_{m}^{k} ; m=1, \ldots, M_{k}\right\}$ are mutually independent and $\left\{\boldsymbol{H}^{k} ; k \in \mathcal{K}_{p}\right\}$ are i.i.d., the asymptotic spectral efficiency of the MIMO DS-CDMA channel with the GPME detector front end is given by

$$
\begin{aligned}
& \mathcal{C}_{\mathrm{sep}}^{\mathrm{STS}}=\beta \lim _{K \rightarrow \infty} \frac{1}{K} \sum_{k=1}^{K} \sum_{m=1}^{M_{k}} \mathcal{C}_{\mathrm{SIMO}, \mathrm{GPME}}^{k, m}(\boldsymbol{R}, \tilde{\boldsymbol{R}}) \quad \text { for STS, } \\
& \mathcal{C}_{\text {sep }}^{\mathrm{TS}}=\beta \lim _{K \rightarrow \infty} \frac{1}{K} \sum_{k=1}^{K} \mathcal{C}_{\mathrm{MIMO}, \mathrm{GPME}}^{k}(\boldsymbol{W}, \tilde{\boldsymbol{W}}) \text { for TS, }
\end{aligned}
$$

where $\mathcal{C}_{\mathrm{SIMO}, \mathrm{GPME}}^{k, m}(\boldsymbol{R}, \tilde{\boldsymbol{R}})$ and $\mathcal{C}_{\mathrm{MIMO}, \mathrm{GPME}}^{k}(\boldsymbol{W}, \tilde{\boldsymbol{W}})$ are the spectral efficiency of the SIMO Gaussian channel (8a) with the GPME detector front end (9a) and the spectral efficiency of the MIMO Gaussian channel (8c) with the GPME detector front end (9b), respectively. $(\boldsymbol{R}, \tilde{\boldsymbol{R}})$ and $(\boldsymbol{W}, \tilde{\boldsymbol{W}})$ are identical to those in Lemma 1,

$\mathcal{C}_{\text {sep }}^{\text {STS }}$ and $\mathcal{C}_{\text {sep }}^{\text {TS }}$ coincide with the first terms of (12a) and (12b), respectively, if $p\left(\boldsymbol{x}^{k}\right)=p\left(\tilde{\boldsymbol{x}}^{k}\right)$ and $N_{0}=\tilde{N}_{0}$, i.e., the first terms represent the spectral efficiencies of the MIMO DS-CDMA channel with the MMSE detector front end in the respective cases.

Although we have used the replica method to derive the results presented so far, the following Corollary demonstrates that Proposition 3 is reduced to the known results with the Gaussian data modulation [12], [17], which have been proved rigorously with random matrix theory.

Corollary 1: If $\left\{x_{m}^{k}, \tilde{x}_{m}^{k} ; m=1, \ldots, M_{k}=M\right\}$ follow i.i.d. $\mathcal{C N}(0, P)$ and if $\left\{\boldsymbol{H}^{k} ; k \in \mathcal{K}\right\}$ are i.i.d. random matrices with i.i.d. entries, then the spectral efficiency of the MIMO DS-CDMA channel with the LMMSE detector front end is given by

$$
\begin{aligned}
\mathcal{C}_{\mathrm{LMMSE}}^{\mathrm{STS}}=\beta M \mathrm{E}\left[\log \left(1+\frac{P\left\|h_{m}^{k}\right\|^{2}}{N_{R}}\right)\right] & \text { for STS, } \\
\mathcal{C}_{\mathrm{LMMSE}}^{\mathrm{TS}}=\beta \mathrm{E}\left[\log \operatorname{det}\left(\boldsymbol{I}_{N}+\frac{P}{N_{W}} \boldsymbol{H}^{k}\left(\boldsymbol{H}^{k}\right)^{H}\right)\right] & \text { for TS, }
\end{aligned}
$$


where $N_{R}$ is the unique solution of the fixed-point equation

$$
N_{R}=N_{0}+\frac{\beta}{N} \mathrm{E}\left[\frac{P\left\|\boldsymbol{h}_{m}^{k}\right\|^{2}}{1+P\left\|\boldsymbol{h}_{m}\right\|^{2} / N_{R}}\right],
$$

and $N_{W}$ is the unique solution of the fixed-point equation

$$
N_{W}=N_{0}+\frac{\beta}{N} \min (N, M) \mathrm{E}\left[\frac{P \lambda}{1+P \lambda / N_{W}}\right]
$$

with $\lambda$ being the random variable following the marginal distribution of the unordered singular values of $\boldsymbol{H}^{k}[19]$.

The above result for the STS scheme is consistent with that reported in [12], [17]. On the other hand, the result for the TS scheme includes and extends the result in [12], the latter of which has been proved only when the number of receive antennas is one or when the number of transmit antennas is infinite.

\section{NumericAl RESUlts}

We compare numerically the spectral efficiency of the MIMO DS-CDMA channel in the case of several transmitter and receiver structures. In this section, we assume that the number of transmit antennas is uniform, i.e., $M_{k}=M$, that $\left\{x_{m}^{k}\right\}$ are i.i.d. random variables with variance $P$, and $\left\{h_{n m}^{k}\right\} \sim$ i.i.d. $\mathcal{C N}(0,1 / N)$. From these assumptions, the spectral efficiencies (12a), (12b), $(22 \mathrm{a})$, and (22b) converge almost surely to their expectations with respect to $\mathcal{H}$. Figure 3 shows the spectral efficiency of the MIMO DS-CDMA channel with the STS scheme and that with the TS scheme, in the case of the Gaussian data modulation. The maximum spectral efficiency of the MIMO DS-CDMA channel with the STS scheme is slightly larger than that with the TS scheme even if optimal space-time encoding is employed. This result implies that the TS scheme is suboptimal. On the other hand, the spectral efficiency of the MIMO DS-CDMA channel with the MMSE detector front end is slightly larger in the case of the TS scheme than in the case of the STS scheme when the system load $\beta$ is high.

We next consider the spectral efficiency of the MIMO DS-CDMA channel in the case in which the data modulation scheme is specified. Figure 4 shows the spectral efficiency of the MIMO DS-CDMA channel with the QPSK data modulation scheme in the case of the STS scheme. The spectral efficiency of the MIMO DS-CDMA channel with the MMSE detector front end displays waterfall behavior in the case of multiple antennas. The spectral efficiency 
is much larger than the spectral efficiency of the MIMO DS-CDMA channel with the LMMSE detector when the system load $\beta$ is smaller than the transition point of the waterfall behavior. Furthermore, the spectral efficiency is comparable to the maximum spectral efficiency of the MIMO DS-CDMA channel, which is very close to the sum capacity when the system load is just below the transition point. However, it decreases rapidly as the system load $\beta$ becomes larger than the transition point.

\section{DISCUSSION}

The results in Section IV imply that the separation loss can be reduced dramatically by employing a non-Gaussian data modulation scheme, even though it has less information per received power than the Gaussian data modulation, and taking the data modulation scheme into consideration in multiuser detection. Although it is difficult to perform the multiuser detection exactly, approximate methods of multiuser detection, e.g., an algorithm on the basis of message passing, may provide higher performance than linear multiuser detectors.

One can obtain, from Propositions 2 and 3, hints about an appropriate structure of the transmitter and a method of constructing multiuser detection algorithms in many-user systems. In the case of the STS scheme, the spectral efficiency of the MIMO DS-CDMA channel with the MMSE detector front end is achieved by per-antenna encoding because (22a) is given by the sum of the spectral efficiencies of the SIMO Gaussian channel $8 \mathrm{a}$ ) with the MMSE detector front end. This means that space-time encoding is not required, and furthermore, that the ergodic spectral efficiency $\mathrm{E}\left[\mathcal{C}_{\text {sep }}^{\mathrm{STS}}\right]$ does not decrease even if the transmit spatial correlation at each user exists. Another suggestion is that one should take a space-time encoded symbol vector $\boldsymbol{x}^{k}$ as a single node in a message passing algorithm for the TS scheme, in order to acquire comparable spectral efficiency to that in the case of the STS scheme, although one can take the element $x_{m}^{k}$ of the symbol vector as a single node for the STS scheme.

Although we have so far considered the uplink of the MIMO DS-CDMA channel, our results can straightforwardly be extended to the cases of an uplink of a frequency-selective MIMO channel with the MC CDMA scheme (MIMO MC-CDMA) [13] and downlinks of these channels.

Our model corresponds to the uplink of the MIMO MC-CDMA channel when $\boldsymbol{H}^{k}$ is replaced by $\boldsymbol{H}_{l}^{k}$, which represents the channel matrix of the $l$ th subcarrier from the $k$ th user to the receiver. Furthermore, (6) coincides with the maximum spectral efficiency per chip and per sub- 
carrier of the MIMO MC-CDMA channel without encoding across the subcarriers if the loss of spectral efficiency due to inserting a cyclic prefix is neglected. If $\left\{\boldsymbol{H}_{l}^{k} ; l=1 \ldots, L\right\}$ are i.i.d., Propositions 1 13, in which $\boldsymbol{H}^{k}$ is replaced by $\boldsymbol{H}_{1}^{k}$, hold for the case in which the ergodic spectral efficiency is considered. On the other hand, our model corresponds to the downlinks of the MIMO DS-CDMA channel and the MIMO MC-CDMA channel if $\left\{\boldsymbol{H}^{k} ; k=1, \ldots, K\right\}$ and $\left\{\boldsymbol{H}_{l}^{k} ; k=1, \ldots, K\right\}$, respectively, represent the same fading [18]. In addition, in this case, Propositions $1-\sqrt{3}$ hold without modification. It is easy to extend these results to the case with encoding across the subcarriers.

\section{CONCLUSION}

We have analyzed the MIMO DS-CDMA channel with the GPME detector front end. In the many-user limit, the MIMO DS-CDMA channel with the MMSE detector front end and the STS scheme is decoupled into a bank of the single-user SIMO Gaussian channels with the MMSE detector front ends. On the other hand, the MIMO DS-CDMA channel with the MMSE detector front end and the TS scheme is decoupled into a bank of the single-user MIMO Gaussian channels with the MMSE detector front end. If suitable space-time encoding is employed, the spectral efficiency of the MIMO DS-CDMA channel with the TS scheme is comparable with the spectral efficiency of the MIMO DS-CDMA channel with the STS scheme, where space-time encoding is not required. Our results suggest huge potentialities of nonlinear multiuser detection, which can exceed linear multiuser detection, and a guideline for methods of constructing message passing algorithms for multiuser detection in many-user systems.

\section{APPENDIX A}

\section{DERIVATION OF PROPOSITION 1}

We define the free energy $\mathcal{F}$ as

$$
\mathcal{F}=-\lim _{K, L \rightarrow \infty} \frac{1}{K} \mathrm{E}\left\{\ln \mathrm{E}_{\overrightarrow{\boldsymbol{x}}}\left[p\left(\overrightarrow{\tilde{\boldsymbol{y}}}=\overrightarrow{\boldsymbol{y}} \mid \overrightarrow{\widetilde{\boldsymbol{x}}}, \mathcal{S}, \mathcal{H} ; \tilde{N}_{0}\right)\right] \mid \boldsymbol{\mathcal { H }}\right\},
$$

where the limit in (26) stands for the many-user limit. From Assumption 4 , in the many-user limit, the maximum spectral efficiency (6) is given by

$$
\lim _{K, L \rightarrow \infty} \mathcal{C}_{\text {joint }}=\left.\frac{\beta}{\ln 2} \mathcal{F}\right|_{p(\overrightarrow{\boldsymbol{x}})=p(\overrightarrow{\boldsymbol{x}}), \tilde{N}_{0}=N_{0}}-N \log \left(\pi N_{0} \mathrm{e}\right) .
$$


We evaluate the free energy (26) using the replica trick. Applying to (26) the identity

$$
\mathrm{E}[\ln Z]=\lim _{u \rightarrow 0} \frac{\partial}{\partial u} \ln \mathrm{E}\left[Z^{u}\right] \quad \text { for } Z>0 \text { a.s., }
$$

we have

$$
\begin{aligned}
\mathcal{F} & =-\lim _{u \rightarrow 0} \frac{\partial}{\partial u} \lim _{K, L \rightarrow \infty} \frac{1}{K} \ln \Xi^{(u)}, \\
\Xi^{(u)} & =\mathrm{E}\left[\left\{\mathrm{E}_{\overrightarrow{\boldsymbol{x}}}\left[p\left(\overrightarrow{\overrightarrow{\boldsymbol{y}}}=\overrightarrow{\boldsymbol{y}} \mid \overrightarrow{\boldsymbol{x}}, \mathcal{S}, \mathcal{H} ; \tilde{N}_{0}\right)\right]\right\}^{u} \mid \mathcal{H}\right],
\end{aligned}
$$

where we have exchanged the order of the many-user limit and the operations with respect to $u$. We evaluate (29b) on $\{u=0,1, \ldots\}$ in the many-user limit, whose domain is extended to the real number field in the neighborhood of $u=0$. The validity of these procedures is pending.

We write the original symbol vector for the $k$ th user and the i.i.d. replicated symbol vectors for the $k$ th user following $p\left(\tilde{\boldsymbol{x}}^{k}\right)$ as $\boldsymbol{x}^{k, 0}$ and $\boldsymbol{x}^{k, \alpha}=\left(x_{1}^{k, \alpha}, \ldots, x_{M_{k}}^{k, \alpha}\right)^{T}$ for $\alpha=1, \ldots, u$, respectively. Due to the independence of the spreading sequences, (29b) yields

$$
\begin{aligned}
\Xi^{(u)} & =\mathrm{E}\left\{\exp \left[L G_{K}^{(u)}(\overrightarrow{\mathcal{X}})\right] \mid \boldsymbol{\mathcal { H }}\right\}, \\
G_{K}^{(u)}(\overrightarrow{\mathcal{X}}) & =\ln \mathrm{E}\left[\int \prod_{\alpha=0}^{u} \exp \left(-\frac{1}{N_{\alpha}}\left\|\boldsymbol{y}-\sqrt{\beta} \boldsymbol{v}^{\alpha}\right\|^{2}\right) \boldsymbol{d} \boldsymbol{y} \mid \overrightarrow{\mathcal{X}}, \mathcal{H}\right]-N \sum_{\alpha=0}^{u} \ln \left(\pi N_{\alpha}\right),
\end{aligned}
$$

where $\overrightarrow{\mathcal{X}}=\left\{\boldsymbol{x}^{k, \alpha} ; k=1, \ldots, K, \alpha=0, \ldots, u\right\}, N_{\alpha}=\tilde{N}_{0}$ for $\alpha=1, \ldots, u$ and $N$-dimensional vector $\boldsymbol{v}^{\alpha}$ is defined as

$$
\boldsymbol{v}^{\alpha}=\frac{1}{\sqrt{\beta}} \sum_{k=1}^{K} \boldsymbol{H}^{k} \boldsymbol{S}^{k} \boldsymbol{x}^{k, \alpha}
$$

with i.i.d. $M_{k} \times M_{k}$ random matrices $\left\{\boldsymbol{S}^{k} ; k=1, \ldots, K\right\}$ following $p\left(\boldsymbol{S}_{l}^{k}\right)$. Since $K$ and $L$ are sufficiently large with their ratio $\beta=K / L$ fixed, due to the central limit theorem, $\boldsymbol{v}=\left(\boldsymbol{v}^{0^{T}}, \ldots, \boldsymbol{v}^{u T}\right)^{T}$ conditioned on $\overrightarrow{\mathcal{X}}$ and $\mathcal{H}$ follows approximately the zero-mean circularly symmetric complex Gaussian distribution with the covariance matrix

$$
\mathcal{Q}=\frac{1}{K} \sum_{k=1}^{K}\left[\delta \boldsymbol{w}^{k}\left(\boldsymbol{w}^{k}\right)^{H}+(1-\delta) \boldsymbol{W}^{k}\left(\boldsymbol{W}^{k}\right)^{H}\right],
$$

where $\boldsymbol{w}^{k}=\left[\left(\boldsymbol{H}^{k} \boldsymbol{x}^{k, 0}\right)^{T} \cdots\left(\boldsymbol{H}^{k} \boldsymbol{x}^{k, u}\right)^{T}\right]^{T}$ and $\boldsymbol{W}^{k}=\left[\left(\boldsymbol{W}^{k, 0}\right)^{T} \cdots\left(\boldsymbol{W}^{k, u}\right)^{T}\right]^{T}$ with $\boldsymbol{W}^{k, \alpha}=$ $\left(\boldsymbol{h}_{1}^{k} x_{1}^{k, \alpha} \cdots \boldsymbol{h}_{M_{k}}^{k} x_{M_{k}}^{k, \alpha}\right)$. Evaluating the Gaussian integration in (30b), we obtain

$$
\begin{aligned}
\frac{1}{K} \ln \Xi^{(u)} & =\frac{1}{K} \ln \mathrm{E}\left\{\exp \left[K \beta^{-1} G^{(u)}(\mathcal{Q})\right] \mid \boldsymbol{H}\right\}+\mathcal{O}\left(K^{-1}\right), \\
G^{(u)}(\mathcal{Q}) & =-\ln \operatorname{det}(\boldsymbol{I}+\boldsymbol{\Sigma} \mathcal{Q})-N u \ln \left(\pi \tilde{N}_{0}\right)-N \ln \left(1+\frac{N_{0}}{\tilde{N}_{0}} u\right),
\end{aligned}
$$


where $\Sigma$ is given by

$$
\boldsymbol{\Sigma}=\frac{\beta}{\tilde{N}_{0}+u N_{0}}\left[\begin{array}{cc}
u & -\boldsymbol{e}_{u}^{T} \\
-\boldsymbol{e}_{u} & \left(1+\frac{u N_{0}}{\tilde{N}_{0}}\right) \boldsymbol{I}_{u}-\frac{N_{0}}{\tilde{N}_{0}} \boldsymbol{e}_{u} \boldsymbol{e}_{u}^{T}
\end{array}\right] \otimes \boldsymbol{I}_{N} .
$$

The expectation with respect to $\overrightarrow{\mathcal{X}}$ in $(33 \mathrm{a})$ is evaluated as the expectation with respect to $\mathcal{Q}$ because (33b) depends on $\overrightarrow{\mathcal{X}}$ only through $\mathcal{Q}$. Since $\mathcal{Q}$ is the empirical mean of independent random variables conditioned on $\mathcal{H}$, the probability density function of $\mathcal{Q}$ conditioned on $\mathcal{H}$ satisfies the large deviation principle

$$
\frac{1}{K} \ln p_{K}^{(u)}(\mathcal{Q} \mid \mathcal{H})=-I^{(u)}(\mathcal{Q})+o(K),
$$

where the rate function $I^{(u)}(\mathcal{Q})$ is given by

$$
I^{(u)}(\mathcal{Q})=\sup _{\tilde{\mathcal{Q}}}\left[\operatorname{Tr}(\tilde{\mathcal{Q}} \mathcal{Q})-\lim _{K \rightarrow \infty} \frac{1}{K} \sum_{k=1}^{K} \ln \mathcal{M}_{k}^{(u)}(\tilde{\mathcal{Q}})\right]
$$

with the moment generating function of the symbols of the $k$ th user $\mathcal{M}_{k}^{(u)}(\tilde{\mathcal{Q}})$ defined as

$$
\mathcal{M}_{k}^{(u)}(\tilde{\mathcal{Q}})=\mathrm{E}\left\{\mathrm{e}^{\Lambda^{k}(\tilde{\mathcal{Q}})} \mid \boldsymbol{H}^{k}\right\}, \quad \Lambda^{k}=\delta \operatorname{Tr}\left(\tilde{\mathcal{Q}} \boldsymbol{w}^{k}\left(\boldsymbol{w}^{k}\right)^{H}\right)+(1-\delta) \operatorname{Tr}\left(\tilde{\mathcal{Q}} \boldsymbol{W}^{k}\left(\boldsymbol{W}^{k}\right)^{H}\right)
$$

Evaluating the expectation in (33a) with the saddle point method, we have

$$
\lim _{K, L \rightarrow \infty} \frac{1}{K} \ln \Xi^{(u)}=\sup _{\mathcal{Q}}\left[\beta^{-1} G^{(u)}(\mathcal{Q})-I^{(u)}(\mathcal{Q})\right]
$$

Setting the derivatives of (36) and (38) with respect to $\tilde{\mathcal{Q}}$ and $Q$, respectively, to zero, we obtain the following equations, which give extrema of (36) and (38):

$$
\begin{aligned}
& \mathcal{Q}^{\mathrm{s}}=\lim _{K \rightarrow \infty} \frac{1}{K} \sum_{k=1}^{K} \frac{1}{\mathcal{M}_{k}^{(u)}\left(\tilde{\mathcal{Q}}^{\mathrm{s}}\right)} \mathrm{E}\left\{\left[\delta \boldsymbol{w}^{k}\left(\boldsymbol{w}^{k}\right)^{H}+(1-\delta) \boldsymbol{W}^{k}\left(\boldsymbol{W}^{k}\right)^{H}\right] \mathrm{e}^{\left.\Lambda^{k}\left(\tilde{\mathcal{Q}}^{\mathrm{s}}\right) \mid \boldsymbol{H}^{k}\right\},}\right. \\
& \tilde{\mathcal{Q}}^{\mathrm{s}}=-\beta^{-1}\left(\boldsymbol{I}+\boldsymbol{\Sigma} \mathcal{Q}^{\mathrm{s}}\right)^{-1} \boldsymbol{\Sigma} .
\end{aligned}
$$

Substituting (39b) into the derivative of (38) with respect to $u$, we can evaluate (29a) as

$$
\mathcal{F}=-\lim _{u \rightarrow 0}\left[\beta^{-1} \frac{\partial G^{(u)}}{\partial u}\left(\mathcal{Q}^{\mathrm{s}}\right)-\frac{\partial I^{(u)}}{\partial u}\left(\mathcal{Q}^{\mathrm{s}}\right)\right] .
$$

We have to solve (39a) and (39b) analytically in order to evaluate (40). In what follows, we assume that the replica symmetry holds 2 , i.e., $\mathcal{Q}^{\mathrm{s}}$ and $\tilde{\mathcal{Q}}^{\mathrm{s}}$ are invariant under the exchange of

\footnotetext{
${ }^{2}$ It should be noted that there exist cases in which the replica symmetry is not valid. See [20] for a discussion of the validity in the context of DS-CDMA.
} 
non-zero replica indexes. Then, $\mathcal{Q}^{\mathrm{s}}$ and $\tilde{\mathcal{Q}}^{\mathrm{s}}$ in the limit $u \rightarrow 0$ can be written as

$$
\begin{aligned}
& \mathcal{Q}^{\mathrm{s}}=\left(\begin{array}{cc}
\boldsymbol{Q}^{0} & \boldsymbol{e}_{u}^{T} \otimes \boldsymbol{M} \\
\boldsymbol{e}_{u} \otimes \boldsymbol{M}^{H} & \boldsymbol{I}_{u} \otimes\left(\boldsymbol{Q}^{1}-\boldsymbol{Q}\right)+\boldsymbol{e}_{u} \boldsymbol{e}_{u}^{T} \otimes \boldsymbol{Q}
\end{array}\right) \\
& \tilde{\mathcal{Q}}^{\mathrm{s}}=\left(\begin{array}{cc}
\tilde{\boldsymbol{Q}}^{0} & \boldsymbol{e}_{u}^{T} \otimes \tilde{\boldsymbol{M}} \\
\boldsymbol{e}_{u} \otimes \tilde{\boldsymbol{M}}^{H} & \boldsymbol{I}_{u} \otimes\left(\tilde{\boldsymbol{Q}}^{1}-\tilde{\boldsymbol{Q}}\right)+\boldsymbol{e}_{u} \boldsymbol{e}_{u}^{T} \otimes \tilde{\boldsymbol{Q}}
\end{array}\right)
\end{aligned}
$$

with $N \times N$ matrices $\boldsymbol{M}, \tilde{\boldsymbol{M}}$ and $N \times N$ Hermitian matrices $\boldsymbol{Q}^{0}, \tilde{\boldsymbol{Q}}^{0}, \boldsymbol{Q}^{1}, \tilde{\boldsymbol{Q}}^{1}, \boldsymbol{Q}$, and $\tilde{\boldsymbol{Q}}$. Comparing both sides of (39b), we obtain in the limit $u \rightarrow 0$

$$
\tilde{\boldsymbol{Q}}^{0}=\mathbf{0}, \quad \tilde{\boldsymbol{M}}=\tilde{\boldsymbol{R}}^{-1}, \quad \tilde{\boldsymbol{Q}}^{1}=\tilde{\boldsymbol{Q}}-\tilde{\boldsymbol{M}}, \quad \tilde{\boldsymbol{Q}}=\tilde{\boldsymbol{R}}^{-1} \boldsymbol{R} \tilde{\boldsymbol{R}}^{-1},
$$

where $\boldsymbol{R}$ and $\tilde{\boldsymbol{R}}$ are respectively given by

$$
\begin{aligned}
\boldsymbol{R} & =N_{0} \boldsymbol{I}_{N}+\beta\left(\boldsymbol{Q}^{0}-\boldsymbol{M}-\boldsymbol{M}^{H}+\boldsymbol{Q}\right), \\
\tilde{\boldsymbol{R}} & =\tilde{N}_{0} \boldsymbol{I}_{N}+\beta\left(\boldsymbol{Q}^{1}-\boldsymbol{Q}\right) .
\end{aligned}
$$

Next, We solve (39a). We describe only the evaluation of the moment generating function (37) because each term of (39a) is evaluated in the same manner. It is straightforward to prove that $\boldsymbol{R}$ is positive definite. Then, one can take a square root of $\boldsymbol{R}$ such that $\boldsymbol{R}=\sqrt{\boldsymbol{R}}(\sqrt{\boldsymbol{R}})^{H}$. Substituting (42) into (37), we obtain

$$
\begin{aligned}
\mathcal{M}_{k}^{(u)}\left(\tilde{\mathcal{Q}}^{\mathrm{s}}\right)= & \mathrm{E}\left\{\operatorname { e x p } \left[\delta\left(\left\|\boldsymbol{b}^{k}\right\|^{2}-\sum_{\alpha=0}^{u}\left(\boldsymbol{H}^{k} \boldsymbol{x}^{k, \alpha}\right)^{H} \boldsymbol{R}_{\alpha}^{-1} \boldsymbol{H}^{k} \boldsymbol{x}^{k, \alpha}\right)\right.\right. \\
& \left.\left.+(1-\delta) \sum_{m=1}^{M_{k}}\left(\left\|\boldsymbol{b}_{m}^{k}\right\|^{2}-\sum_{\alpha=0}^{u}\left(\boldsymbol{h}_{m}^{k} x_{m}^{k, \alpha}\right)^{H} \boldsymbol{R}_{\alpha}^{-1} \boldsymbol{h}_{m}^{k} x_{m}^{k, \alpha}\right)\right] \mid \boldsymbol{H}^{k}\right\},
\end{aligned}
$$

where $\boldsymbol{R}_{0}=\boldsymbol{R}, \boldsymbol{R}_{\alpha}=\tilde{\boldsymbol{R}}$ for $\alpha=1, \ldots, u$ and $\boldsymbol{b}^{k}$ and $\boldsymbol{b}_{m}^{k}$ are given by

$$
\begin{aligned}
& \boldsymbol{b}^{k}=(\sqrt{\boldsymbol{R}})^{-1} \boldsymbol{H}^{k} \boldsymbol{x}^{k, 0}+(\sqrt{\boldsymbol{R}})^{H} \tilde{\boldsymbol{R}}^{-1} \sum_{\alpha=1}^{u} \boldsymbol{H}^{k} \boldsymbol{x}^{k, \alpha}, \\
& \boldsymbol{b}_{m}^{k}=(\sqrt{\boldsymbol{R}})^{-1} \boldsymbol{h}_{m}^{k} x_{m}^{k, 0}+(\sqrt{\boldsymbol{R}})^{H} \tilde{\boldsymbol{R}}^{-1} \sum_{\alpha=1}^{u} \boldsymbol{h}_{m}^{k} x_{m}^{k, \alpha} .
\end{aligned}
$$

Substituting two transforms that are similar to the Hubbard-Stratonovich transform, as follows:

$$
\begin{aligned}
\mathrm{e}^{\delta \mid \boldsymbol{b}^{k} \|^{2}} & =\int \frac{1}{\pi^{N} \operatorname{det}\left(\delta^{-1} \boldsymbol{R}\right)} \mathrm{e}^{\delta\left[-\left(\boldsymbol{y}^{k}\right)^{H} \boldsymbol{R}^{-1} \boldsymbol{y}^{k}+2 \operatorname{Re}\left(\left(\boldsymbol{b}^{k}\right)^{H}(\sqrt{\boldsymbol{R}})^{-1} \boldsymbol{y}^{k}\right)\right]} \boldsymbol{d} \boldsymbol{y}^{k}, \\
\mathrm{e}^{(1-\delta)\left\|\boldsymbol{b}_{m}^{k}\right\|^{2}} & =\int \frac{1}{\pi^{N} \operatorname{det}\left[(1-\delta)^{-1} \boldsymbol{R}\right]} \mathrm{e}^{(1-\delta)\left[-\left(\boldsymbol{y}_{m}^{k}\right)^{H} \boldsymbol{R}^{-1} \boldsymbol{y}_{m}^{k}+2 \operatorname{Re}\left(\left(\boldsymbol{b}_{m}^{k}\right)^{H}(\sqrt{\boldsymbol{R}})^{-1} \boldsymbol{y}_{m}^{k}\right)\right]} \boldsymbol{d} \boldsymbol{y}_{m}^{k},
\end{aligned}
$$


into (44), we have

$$
\mathcal{M}_{k}^{(u)}\left(\tilde{\mathcal{Q}}^{\mathrm{s}}\right)=\int \mathrm{E}_{\boldsymbol{x}^{k}}\left[q\left(\boldsymbol{y}^{k},\left\{\boldsymbol{y}_{m}^{k}\right\} \mid \boldsymbol{x}^{k}, \boldsymbol{H}^{k} ; \boldsymbol{R}\right)\right]\left\{\frac{\mathrm{E}_{\tilde{\boldsymbol{x}}^{k}}\left[q\left(\boldsymbol{y}^{k},\left\{\boldsymbol{y}_{m}^{k}\right\} \mid \tilde{\boldsymbol{x}}^{k}, \boldsymbol{H}^{k} ; \tilde{\boldsymbol{R}}\right)\right]}{q\left(\boldsymbol{y}^{k},\left\{\boldsymbol{y}_{m}^{k}\right\} \mid \mathbf{0}, \boldsymbol{H}^{k} ; \tilde{\boldsymbol{R}}\right)}\right\}^{u} \boldsymbol{d} \boldsymbol{y}^{k} \prod_{m=1}^{M_{k}} \boldsymbol{d} \boldsymbol{y}_{m}^{k}
$$

where the function $q\left(\boldsymbol{y}^{k},\left\{\boldsymbol{y}_{m}^{k}\right\} \mid \boldsymbol{x}^{k}, \boldsymbol{H}^{k} ; \boldsymbol{R}\right)$ is given by

$$
\begin{aligned}
& q\left(\boldsymbol{y}^{k},\left\{\boldsymbol{y}_{m}^{k}\right\} \mid \boldsymbol{x}^{k}, \boldsymbol{H}^{k} ; \boldsymbol{R}\right)=q\left(\boldsymbol{y}^{k} \mid \boldsymbol{x}^{k}, \boldsymbol{H}^{k} ; \delta^{-1} \boldsymbol{R}\right) \prod_{m=1}^{M_{k}} p\left(\boldsymbol{y}_{m}^{k} \mid x_{m}^{k}, \boldsymbol{h}_{m}^{k} ;(1-\delta)^{-1} \boldsymbol{R}\right), \\
& q\left(\boldsymbol{y}^{k} \mid \boldsymbol{x}^{k}, \boldsymbol{H}^{k} ; \boldsymbol{R}\right)=\frac{1}{\pi^{N} \operatorname{det} \boldsymbol{R}} \exp \left[-\left(\boldsymbol{y}^{k}-\boldsymbol{H}^{k} \boldsymbol{x}^{k}\right)^{H} \boldsymbol{R}^{-1}\left(\boldsymbol{y}^{k}-\boldsymbol{H}^{k} \boldsymbol{x}^{k}\right)\right] \\
& q\left(\boldsymbol{y}_{m}^{k} \mid x_{m}^{k}, \boldsymbol{h}_{m}^{k} ; \boldsymbol{R}\right)=\frac{1}{\pi^{N} \operatorname{det} \boldsymbol{R}} \exp \left[-\left(\boldsymbol{y}_{m}^{k}-\boldsymbol{h}_{m}^{k} x_{m}^{k}\right)^{H} \boldsymbol{R}^{-1}\left(\boldsymbol{y}_{m}^{k}-\boldsymbol{h}_{m}^{k} x_{m}^{k}\right)\right]
\end{aligned}
$$

Evaluating (39a) in the same manner and substituting the results into (43a) and (43b), we obtain the following fixed-point equations:

$$
\begin{aligned}
& \boldsymbol{R}=N_{0} \boldsymbol{I}_{N}+\beta \lim _{K \rightarrow \infty} \frac{1}{K} \sum_{k=1}^{K}\left[\delta \boldsymbol{H}^{k} \mathcal{E}_{\mathrm{MIMO}}^{k, \delta}\left(\boldsymbol{H}^{k}\right)^{H}+(1-\delta) \sum_{m=1}^{M_{k}} \mathcal{E}_{\mathrm{SIMO}}^{k, m, \delta} \boldsymbol{h}_{m}^{k}\left(\boldsymbol{h}_{m}^{k}\right)^{H}\right], \\
& \tilde{\boldsymbol{R}}=\tilde{N}_{0} \boldsymbol{I}_{N}+\beta \lim _{K \rightarrow \infty} \frac{1}{K} \sum_{k=1}^{K}\left[\delta \boldsymbol{H}^{k} \mathcal{V}_{\mathrm{MIMO}}^{k, \delta}\left(\boldsymbol{H}^{k}\right)^{H}+(1-\delta) \sum_{m=1}^{M_{k}} \mathcal{V}_{\mathrm{SIMO}}^{k, m, \delta} \boldsymbol{h}_{m}^{k}\left(\boldsymbol{h}_{m}^{k}\right)^{H}\right]
\end{aligned}
$$

with $\mathcal{E}_{\mathrm{MIMO}}^{k, \delta}, \mathcal{E}_{\mathrm{SIMO}}^{k, \delta}, \mathcal{V}_{\mathrm{MIMO}}^{k, m, \delta}$, and $\mathcal{V}_{\mathrm{SIMO}}^{k, m, \delta}$ defined respectively as

$$
\begin{aligned}
\mathcal{E}_{\mathrm{MIMO}}^{k, \delta} & =\mathrm{E}\left[\left(\boldsymbol{x}^{k}-\left\langle\tilde{\boldsymbol{x}}^{k}\right\rangle_{\mathrm{s}}\right)\left(\boldsymbol{x}^{k}-\left\langle\tilde{\boldsymbol{x}}^{k}\right\rangle_{\mathrm{s}}\right)^{H} \mid \boldsymbol{H}^{k}\right], \\
\mathcal{E}_{\mathrm{SIMO}}^{k, m, \delta} & =\mathrm{E}\left[\left|x_{m}^{k}-\left\langle\tilde{x}_{m}^{k}\right\rangle_{\mathrm{s}}\right|^{2} \mid \boldsymbol{h}_{m}^{k}\right], \\
\mathcal{V}_{\text {MIMO }}^{k, \delta} & =\mathrm{E}\left[\left(\tilde{\boldsymbol{x}}^{k}-\left\langle\tilde{\boldsymbol{x}}^{k}\right\rangle_{\mathrm{s}}\right)\left(\tilde{\boldsymbol{x}}^{k}-\left\langle\tilde{\boldsymbol{x}}^{k}\right\rangle_{\mathrm{s}}\right)^{H} \mid \boldsymbol{H}^{k}\right], \\
\mathcal{V}_{\mathrm{SIMO}}^{k, m, \delta} & =\mathrm{E}\left[\left|\tilde{x}_{m}^{k}-\left\langle\tilde{x}_{m}^{k}\right\rangle_{\mathrm{s}}\right|^{2} \mid \boldsymbol{h}_{m}^{k}\right],
\end{aligned}
$$

where the expectations are taken with respect to $q\left(\boldsymbol{y}^{k},\left\{\boldsymbol{y}_{m}^{k}\right\} \mid \boldsymbol{x}^{k}, \boldsymbol{H}^{k} ; \boldsymbol{R}\right) p\left(\boldsymbol{x}^{k}\right)$ and $\left\langle\tilde{\boldsymbol{x}}^{k}\right\rangle_{\mathrm{S}}$ is given by

$$
\left\langle\tilde{\boldsymbol{x}}^{k}\right\rangle_{\mathrm{s}}=\frac{\mathrm{E}_{\tilde{\boldsymbol{x}}^{k}}\left[\tilde{\boldsymbol{x}}^{k} q\left(\boldsymbol{y}^{k},\left\{\boldsymbol{y}_{m}^{k}\right\} \mid \tilde{\boldsymbol{x}}^{k}, \boldsymbol{H}^{k} ; \tilde{\boldsymbol{R}}\right)\right]}{\mathrm{E}_{\tilde{\boldsymbol{x}}^{k}}\left[q\left(\boldsymbol{y}^{k},\left\{\boldsymbol{y}_{m}^{k}\right\} \mid \tilde{\boldsymbol{x}}^{k}, \boldsymbol{H}^{k} ; \tilde{\boldsymbol{R}}\right)\right]} .
$$

Since (52) is reduced to (9a) and (9b) for $\delta \rightarrow 0$ and $\delta \rightarrow 1$, respectively, (50a) and (50b) yield (16a) and (16b), and (17a) and (17b) for $\delta \rightarrow 0$ and $\delta \rightarrow 1$, respectively. 
Differentiating the rate function (36) with respect to $u$, we obtain

$$
\lim _{u \rightarrow 0} \frac{\partial I^{(u)}}{\partial u}\left(\mathcal{Q}^{\mathrm{s}}\right)=\lim _{K \rightarrow \infty} \frac{1}{K} \sum_{k=1}^{K} \tilde{\mathcal{C}}_{\mathrm{s}}^{k} \ln 2-\frac{1}{\beta} \operatorname{Tr}\left[\boldsymbol{I}_{N}-\left(N_{0}+\tilde{N}_{0}\right) \tilde{\boldsymbol{R}}^{-1}+\tilde{N}_{0} \tilde{\boldsymbol{R}}^{-1} \boldsymbol{R} \tilde{\boldsymbol{R}}^{-1}\right]
$$

where $\tilde{\mathcal{C}}_{\mathrm{s}}^{k}$ is given by

$$
\tilde{\mathcal{C}}_{\mathrm{s}}^{k}=\mathrm{E}_{\boldsymbol{x}^{k}}\left\{\int q\left(\boldsymbol{y}^{k},\left\{\boldsymbol{y}_{m}^{k}\right\} \mid \boldsymbol{x}^{k}, \boldsymbol{H}^{k} ; \boldsymbol{R}\right) \log \frac{q\left(\boldsymbol{y}^{k},\left\{\boldsymbol{y}_{m}^{k}\right\} \mid \boldsymbol{x}^{k}, \boldsymbol{H}^{k} ; \tilde{\boldsymbol{R}}\right)}{\mathrm{E}_{\tilde{\boldsymbol{x}}^{k}}\left[q\left(\boldsymbol{y}^{k},\left\{\boldsymbol{y}_{m}^{k}\right\} \mid \tilde{\boldsymbol{x}}^{k}, \boldsymbol{H}^{k} ; \tilde{\boldsymbol{R}}\right)\right]} \boldsymbol{d} \boldsymbol{y}^{k} \prod_{m=1}^{M_{k}} \boldsymbol{d} \boldsymbol{y}_{m}^{k}\right\} .
$$

On the other hand, 33b yields

$$
G^{(u)}\left(\mathcal{Q}^{\mathrm{s}}\right)=-(u-1) \ln \operatorname{det} \tilde{\boldsymbol{R}}-\ln \operatorname{det}(\tilde{\boldsymbol{R}}+u \boldsymbol{R})-u N \ln \pi .
$$

Differentiating (55) with respect to $u$, we obtain

$$
\lim _{u \rightarrow 0} \frac{\partial G^{(u)}}{\partial u}=-\ln \operatorname{det} \tilde{\boldsymbol{R}}-\operatorname{Tr}\left(\boldsymbol{R} \tilde{\boldsymbol{R}}^{-1}\right)-N \ln \pi .
$$

From (53) and (56), the free energy (40) is evaluated as

$$
\frac{\beta}{\ln 2} \mathcal{F}=\lim _{K \rightarrow \infty} \frac{1}{K} \sum_{k=1}^{K} \beta \tilde{\mathcal{C}}_{\mathrm{s}}^{k}+N \log \left(\pi N_{0} \mathrm{e}\right)+F(\boldsymbol{R}, \tilde{\boldsymbol{R}})
$$

where $F(\boldsymbol{R}, \tilde{\boldsymbol{R}})$ is given by (20). In particular, (157) is reduced to (18a) and (18b) for $\delta \rightarrow 0$ and $\delta \rightarrow 1$, respectively. In the case in which there exist multiple solutions of (50a) and (50b), one should choose the solution that yields the supremum of (38), which minimizes the free energy (57).

\section{APPENDIX B}

\section{DERIVATION OF LEMMA 1}

With mutually disjoint subsets $A_{m}^{(\mathrm{r})}$ and $A_{m}^{(\mathrm{i})}$ of $\mathcal{A}=\{1, \ldots, u\}$ satisfying $\left|A_{m}^{(\mathrm{r})}\right|=j_{m}^{(\mathrm{r})}$ and $\left|A_{m}^{(\mathrm{i})}\right|=j_{m}^{(\mathrm{i})}$, and with nonnegative integers $i_{m}^{(\mathrm{r})}$ and $i_{m}^{(\mathrm{i})}$, we define a scalar function $f_{k}$ as

$$
f_{k}(\overrightarrow{\mathcal{X}})=\prod_{m=1}^{M_{k}}\left\{\left[\operatorname{Re}\left(x_{m}^{k, 0}\right)\right]_{m}^{i_{m}^{(\mathrm{r})}}\left[\operatorname{Im}\left(x_{m}^{k, 0}\right)\right]_{m}^{i_{m}^{(\mathrm{i})}} \prod_{\alpha_{m}^{(\mathrm{r})} \in A_{m}^{(\mathrm{r})}} \operatorname{Re}\left(x_{m}^{k, \alpha_{m}^{(\mathrm{r})}}\right) \prod_{\alpha_{m}^{(\mathrm{i})} \in A_{m}^{(\mathrm{i})}} \operatorname{Im}\left(x_{m}^{k, \alpha_{m}^{(\mathrm{i})}}\right)\right\} .
$$

Furthermore, with a real parameter $\omega$, we define a quantity similar to the free energy as

$$
\tilde{\mathcal{F}}=\lim _{K, L \rightarrow \infty} \frac{1}{K} \ln \tilde{\Xi}^{(u)}(\omega),
$$

where $\tilde{\Xi}^{(u)}(\omega)$ is given by

$$
\tilde{\Xi}^{(u)}(\omega)=\mathrm{E}\left\{\exp \left[\omega \sum_{k \in \mathcal{K}_{p}} f_{k}(\overrightarrow{\boldsymbol{\mathcal { X }}})\right] \prod_{\alpha=1}^{u} p\left(\overrightarrow{\overrightarrow{\boldsymbol{y}}}=\overrightarrow{\boldsymbol{y}} \mid \overrightarrow{\boldsymbol{x}}^{\alpha}, \mathcal{S}, \mathcal{H} ; \tilde{N}_{0}\right) \mid \mathcal{H}\right\} .
$$


Differentiating (59) with respect to $\omega$, we obtain

$$
\left.\lim _{u \rightarrow 0} \frac{\partial \tilde{\mathcal{F}}}{\partial \omega}\right|_{\omega=0}=\lim _{u \rightarrow 0} \lim _{K, L \rightarrow \infty} \frac{1}{K} \sum_{k \in \mathcal{K}_{p}} \mathrm{E}\left\{f_{k}(\overrightarrow{\boldsymbol{\mathcal { X }}}) \prod_{\alpha=1}^{u} p\left(\overrightarrow{\tilde{\boldsymbol{y}}}=\overrightarrow{\boldsymbol{y}} \mid \overrightarrow{\boldsymbol{x}}^{\alpha}, \mathcal{S}, \mathcal{H} ; \tilde{N}_{0}\right) \mid \mathcal{H}\right\} .
$$

Substituting the Bayes formula

$$
p\left(\overrightarrow{\overrightarrow{\boldsymbol{y}}} \mid \overrightarrow{\boldsymbol{x}}^{\alpha}, \mathcal{S}, \mathcal{H} ; \tilde{N}_{0}\right)=\frac{p\left(\overrightarrow{\boldsymbol{x}}^{\alpha} \mid \overrightarrow{\overrightarrow{\boldsymbol{y}}}, \mathcal{S}, \mathcal{H} ; \tilde{N}_{0}\right) \mathrm{E}_{\overrightarrow{\boldsymbol{x}}}\left[p\left(\overrightarrow{\tilde{\boldsymbol{y}}} \mid \overrightarrow{\overrightarrow{\boldsymbol{x}}}, \mathcal{S}, \mathcal{H} ; \tilde{N}_{0}\right)\right]}{p\left(\overrightarrow{\boldsymbol{x}}^{\alpha}\right)}
$$

into (61), we obtain

$$
\left.\lim _{u \rightarrow 0} \frac{\partial \tilde{\mathcal{F}}}{\partial \omega}\right|_{\omega=0}=\lim _{K, L \rightarrow \infty} \frac{1}{K} \sum_{k \in \mathcal{K}_{p}} \mathrm{E}\left[\prod_{m=1}^{M_{k}}\left\{\left[\operatorname{Re}\left(x_{m}^{k}\right)\right]^{i_{m}^{(\mathrm{r})}}\left[\operatorname{Im}\left(x_{m}^{k}\right)\right]^{i_{m}^{(\mathrm{i})}}\left\langle\operatorname{Re}\left(\tilde{x}_{m}^{k}\right)\right\rangle^{j_{m}^{(\mathrm{r})}}\left\langle\operatorname{Im}\left(\tilde{x}_{m_{k}}^{k}\right)\right\rangle^{j_{m}^{(\mathrm{i})}}\right\} \mid \mathcal{H}\right],
$$

where we have assumed that the limit with respect to $u$ and the many-user limit can be interchanged.

Next, we evaluate (59). We obtain the following expression corresponding to (38):

$$
\tilde{\mathcal{F}}=\sup _{\mathcal{Q}}\left[\beta^{-1} G^{(u)}(\mathcal{Q})-I^{(u)}(\mathcal{Q} ; \omega)\right]
$$

where the extended rate function $I^{(u)}(\mathcal{Q} ; \omega)$ is given by

$$
I^{(u)}(\mathcal{Q} ; \omega)=\sup _{\tilde{\mathcal{Q}}}\left[\operatorname{Tr}(\tilde{\mathcal{Q}} \mathcal{Q})-\lim _{K \rightarrow \infty} \frac{1}{K}\left(\sum_{k \notin \mathcal{K}_{p}} \ln \mathcal{M}_{k}^{(u)}(\tilde{\mathcal{Q}})+\ln \tilde{\mathcal{M}}^{(u)}(\tilde{\mathcal{Q}} ; \omega)\right)\right]
$$

with the extended moment generating function of the symbols defined as

$$
\tilde{\mathcal{M}}^{(u)}(\tilde{\mathcal{Q}} ; \omega)=\mathrm{E}\left\{\exp \left[\sum_{k \in \mathcal{K}_{p}}\left(\omega f_{k}(\overrightarrow{\mathcal{X}})+\Lambda^{k}(\tilde{\mathcal{Q}})\right)\right] \mid \mathcal{H}\right\}
$$

Differentiating $\tilde{\mathcal{F}}$ with respect to $\omega$, we obtain

$$
\left.\lim _{u \rightarrow 0} \frac{\partial \tilde{\mathcal{F}}}{\partial \omega}\right|_{\omega=0}=-\left.\lim _{u \rightarrow 0} \frac{\partial I^{(u)}}{\partial \omega}\left(\mathcal{Q}^{\mathrm{s}} ; \omega\right)\right|_{\omega=0},
$$

where $\mathcal{Q}^{\text {s }}$ satisfies (39a) and (39b).

Assuming the replica symmetry, we can evaluate (67) analytically. The derivative of the extended moment generating function $\tilde{\mathcal{M}}^{(u)}\left(\tilde{\mathcal{Q}}^{\text {s }} ; \omega\right)$ with respect to $\omega$ is given by

$$
\begin{aligned}
\left.\frac{\partial \tilde{\mathcal{M}}^{(u)}}{\partial \omega}\left(\tilde{\mathcal{Q}}^{\mathrm{s}} ; \omega\right)\right|_{\omega=0} & =\sum_{k \in \mathcal{K}_{p}} \int \mathrm{E}_{\boldsymbol{x}^{k}}\left[\prod_{m=1}^{M_{k}}\left\{\left[\operatorname{Re}\left(x_{m}^{k}\right)\right]_{m}^{i_{m}^{(\mathrm{r})}}\left[\operatorname{Im}\left(x_{m}^{k}\right)\right]^{i_{m}^{(\mathrm{i})}}\right\} q\left(\boldsymbol{y}^{k},\left\{\boldsymbol{y}_{m}^{k}\right\} \mid \boldsymbol{x}^{k}, \boldsymbol{H}^{k} ; \boldsymbol{R}\right)\right] \\
& \prod_{m=1}^{M_{k}}\left\{\left\langle\operatorname{Re}\left(\tilde{x}_{m}^{k}\right)\right\rangle_{\mathrm{s}}^{j_{m}^{(\mathrm{r})}}\left\langle\operatorname{Im}\left(\tilde{x}_{m_{k}}^{k}\right)\right\rangle_{\mathrm{s}}^{j_{m}^{(\mathrm{i})}}\right\}\left\{\frac{\mathrm{E}_{\tilde{\boldsymbol{x}}^{k}}\left[q\left(\boldsymbol{y}^{k},\left\{\boldsymbol{y}_{m}^{k}\right\} \mid \tilde{\boldsymbol{x}}^{k}, \boldsymbol{H}^{k} ; \tilde{\boldsymbol{R}}\right)\right]}{q\left(\boldsymbol{y}^{k},\left\{\boldsymbol{y}_{m}^{k}\right\} \mid \mathbf{0}, \boldsymbol{H}^{k} ; \tilde{\boldsymbol{R}}\right)}\right\} \boldsymbol{d y}^{k} \prod_{m=1}^{M_{k}} \boldsymbol{d} \boldsymbol{y}_{m}^{k},(68)
\end{aligned}
$$


where $\boldsymbol{R}$ and $\tilde{\boldsymbol{R}}$ satisfy (50a) and (50b). From (63), (65), (67), and (68), we obtain

$$
\begin{aligned}
& \lim _{K, L \rightarrow \infty} \frac{1}{\left|\mathcal{K}_{p}\right|} \sum_{k \in \mathcal{K}_{p}} \mathrm{E}\left[\prod_{m=1}^{M_{k}}\left\{\left[\operatorname{Re}\left(x_{m}^{k}\right)\right]^{i_{m}^{(\mathrm{r})}}\left[\operatorname{Im}\left(x_{m}^{k}\right)\right]^{i_{m}^{(\mathrm{i})}}\left\langle\operatorname{Re}\left(\tilde{x}_{m}^{k}\right)\right\rangle^{j_{m}^{(\mathrm{r})}}\left\langle\operatorname{Im}\left(\tilde{x}_{m_{k}}^{k}\right)\right\rangle^{j_{m}^{(\mathrm{i})}}\right\} \mid \mathcal{H}\right] \\
= & \lim _{\left|\mathcal{K}_{p}\right| \rightarrow \infty} \frac{1}{\left|\mathcal{K}_{p}\right|} \sum_{k \in \mathcal{K}_{p}} \mathrm{E}\left[\prod_{m=1}^{M_{k}}\left\{\left[\operatorname{Re}\left(x_{m}^{k}\right)\right]^{i_{m}^{(\mathrm{r})}}\left[\operatorname{Im}\left(x_{m}^{k}\right)\right]^{i_{m}^{(\mathrm{i})}}\left\langle\operatorname{Re}\left(\tilde{x}_{m}^{k}\right)\right\rangle_{\mathrm{s}}^{j_{m}^{(\mathrm{r})}}\left\langle\operatorname{Im}\left(\tilde{x}_{m}^{k}\right)\right\rangle_{\mathrm{s}}^{j_{m}^{(\mathrm{i})}}\right\} \mid \boldsymbol{H}^{k}\right] .
\end{aligned}
$$

\section{ACKNOWLEDGMENT}

\section{TT acknowledges support through Grant-in-Aid for Scientific Research on Priority Areas (No.} 18079010) from MEXT, Japan.

\section{REFERENCES}

[1] S. Glisic, Advanced Wireless Communications 4G Technologies. John Wiley \& Sons Inc, 2004.

[2] H. Bölcskei, D. Gesbert, and A. J. Paulraj, "On the capacity of OFDM-based spatial multiplexing systems," IEEE Trans. Commun., vol. 50, pp. 225-234, Feb. 2002.

[3] S. Visuri and H. Bölcskei, "Multiple-access strategies for frequency-selective MIMO channels," IEEE Trans. Inf. Theory, vol. 52, pp. 3980-3993, Sep. 2006.

[4] H. Jafarkhani, Space-Time-Coding Theory and Practice. Cambridge, 2005, ch. 4.

[5] R. G. Gallager, "An inequality on the capacity region of multiaccess multipath channels," in Communications and Cryptography: Two Sides of One Tapestry, R. E. Blahut, D. J. Costello, U. Maurer, and T. Mittelholzer, Eds. New York: Kluwer, 1994, pp. 129-139.

[6] S. Verdú and S. Shamai, "Spectral efficiency of CDMA with random spreading," IEEE Trans. Inf. Theory, vol. 45, pp. 622-640, Mar. 1999.

[7] D. N. C. Tse and S. V. Hanly, "Linear multiuser receivers: effective interference, effective bandwidth and user capacity," IEEE Trans. Inf. Theory, vol. 45, pp. 641-657, Mar. 1999.

[8] T. Tanaka, "A statistical-mechanics approach to large-system analysis of CDMA multiuser detectors," IEEE Trans. Inf. Theory, vol. 48, pp. 2888-2910, Nov. 2002.

[9] D. Guo and S. Verdú, "Randomly spread CDMA: Asymptotics via statistical physics," IEEE Trans. Inf. Theory, vol. 51, pp. 1983-2010, Jun. 2005.

[10] Y. Kabashima, "A CDMA multiuser detection algorithm on the basis of belief propagation," J. Phys. A: Math. Gen., vol. 36, pp. $11111-11121,2003$.

[11] T. Ikehara and T. Tanaka, "Decoupling principle in belief-propagation-based CDMA multiuser detection algorithm," to be published in Proc. 2007 IEEE Int. Symp. Inf. Theory, Nice, France, Jun. 2007.

[12] A. Mantravadi, V. V. Veeravalli, and H. Viswanathan, "Spectral efficiency of MIMO multiaccess systems with single-user decoding," IEEE J. Sel. Areas Commun., vol. 21, pp. 382-394, Apr. 2003.

[13] M. Juntti, J. L. M. Vehkapera, Z. Li, D. Tujkovic, S. Tsumura, and S. Hara, "MIMO MC-CDMA communications for future cellular systems," IEEE Commun. Mag., vol. 43, pp. 118-124, Feb. 2005.

[14] Z. Ni and D. Li, "Spectral efficiency of distributed MIMO code division multiple access systems over multipath fading channels," Wirel. Commun. Mob. Comput., vol. 5, pp. 35-43, 2005. 
[15] A. Nordio and G. Taricco, "Linear receivers for the multiple-input multiple-output multiple-access channel," IEEE Trans. Commun., vol. 54, pp. 1446-1456, Aug. 2006.

[16] D. N. C. Tse and P. Viswanath, Fundamentals of Wireless Communication. Cambridge University Press, 2005 , ch. 8.

[17] S. V. Hanly and D. N. C. Tse, "Resource pooling and effective bandwidths in CDMA networks with multiuser receivers and spatial diversity," IEEE Trans. Inform. Theory, vol. 47, pp. 1328-1351, May 2001.

[18] D. Guo, "Performance of multicarrier CDMA in frequency-selective fading via statistical physics," IEEE Trans. Inf. Theory, vol. 52, pp. 1765-1774, Apr. 2006.

[19] A. M. Tulino and S. Verdú, Random Matrix Theory and Wireless Communications. Now Publishers Inc., 2004 , ch. 2.

[20] M. Yoshida, T. Uezu, T. Tanaka, and M. Okada, "Statistical mechanical study of code-division multiple-access multiuser detectors," J. Phys. Soc. Jpn., vol. 76, pp. 054003-1-10, 2007. 


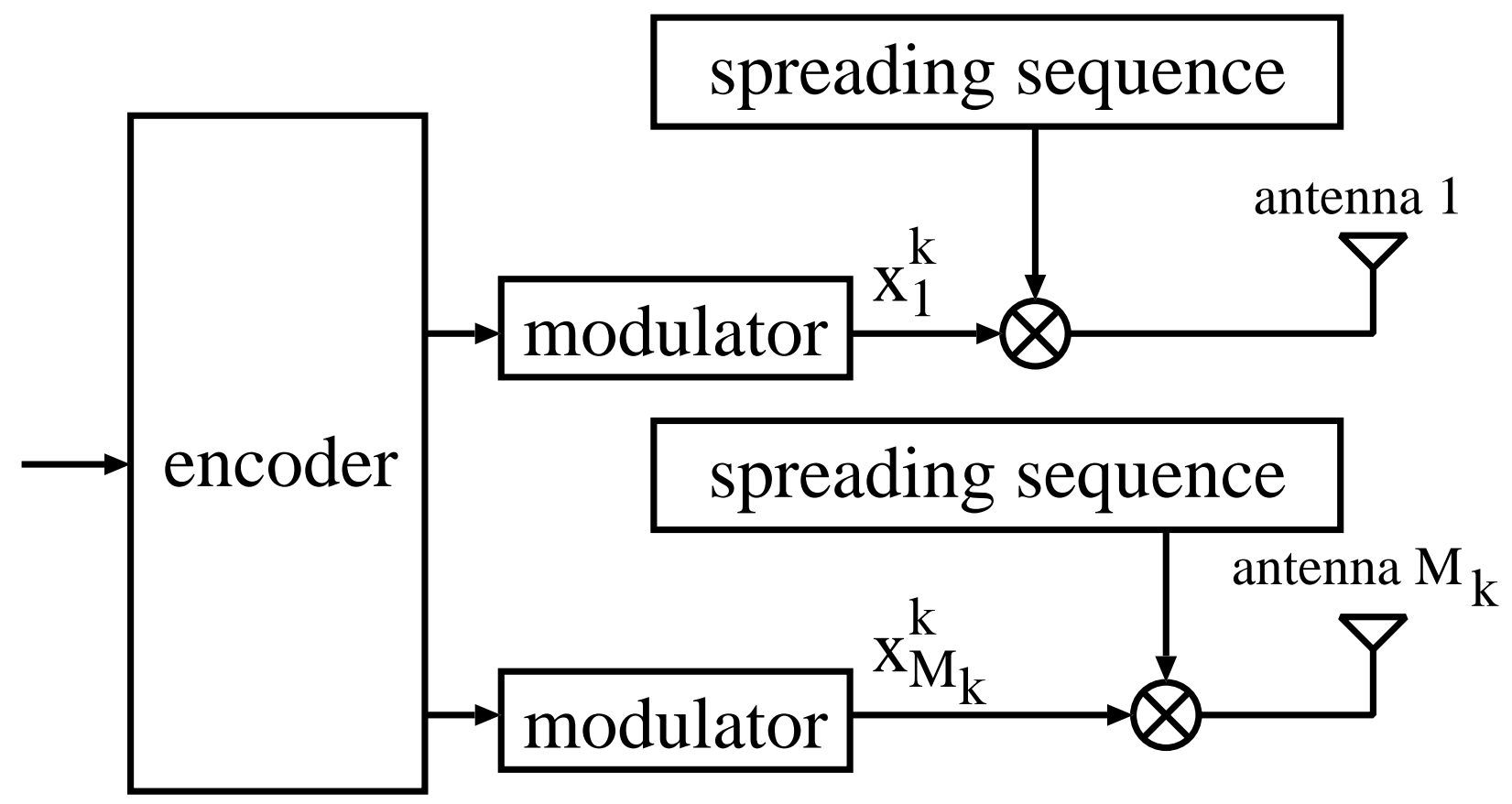

Fig. 1. Transmitter structure with the STS scheme. 


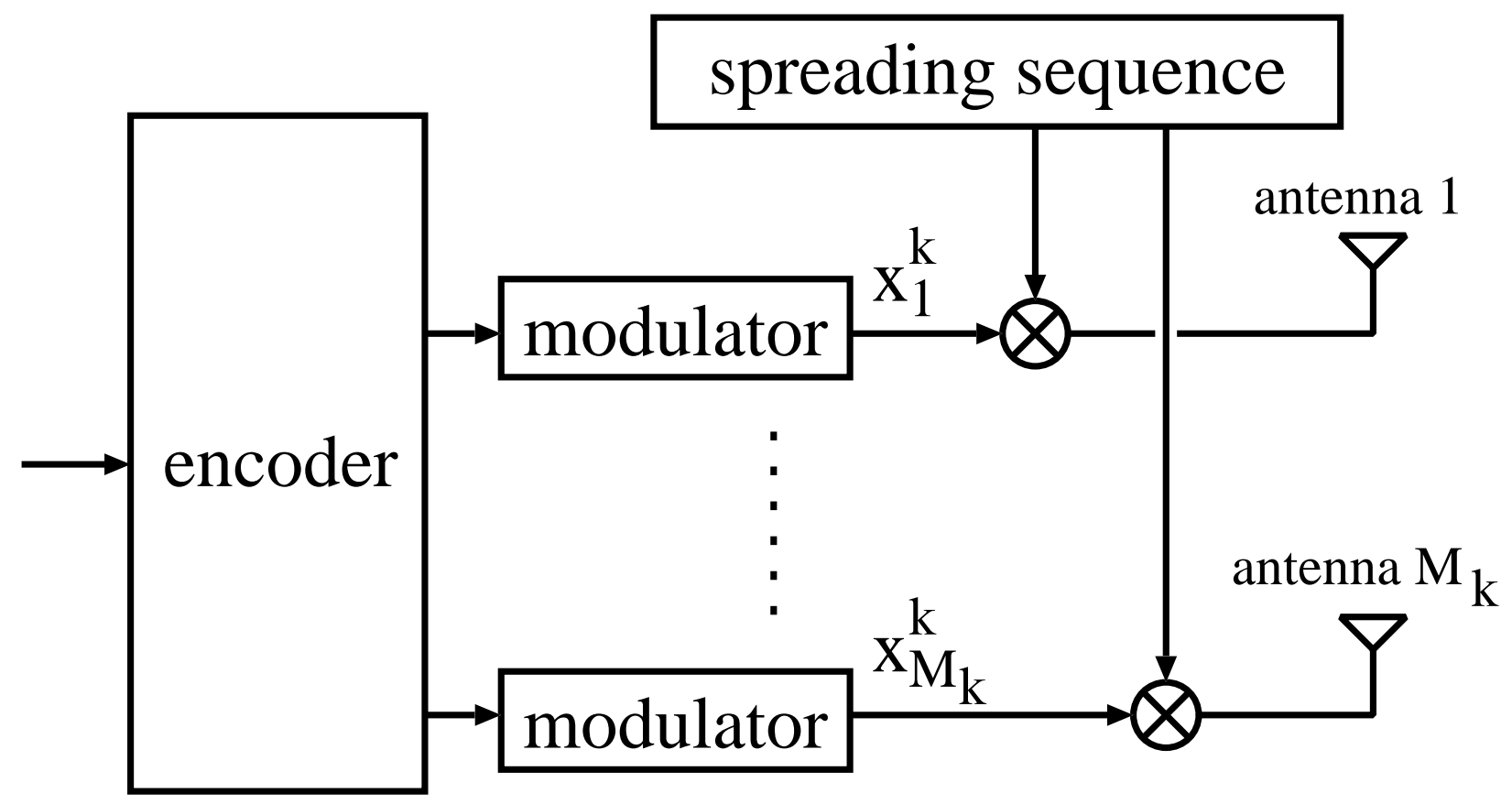

Fig. 2. Transmitter structure with the TS scheme. 


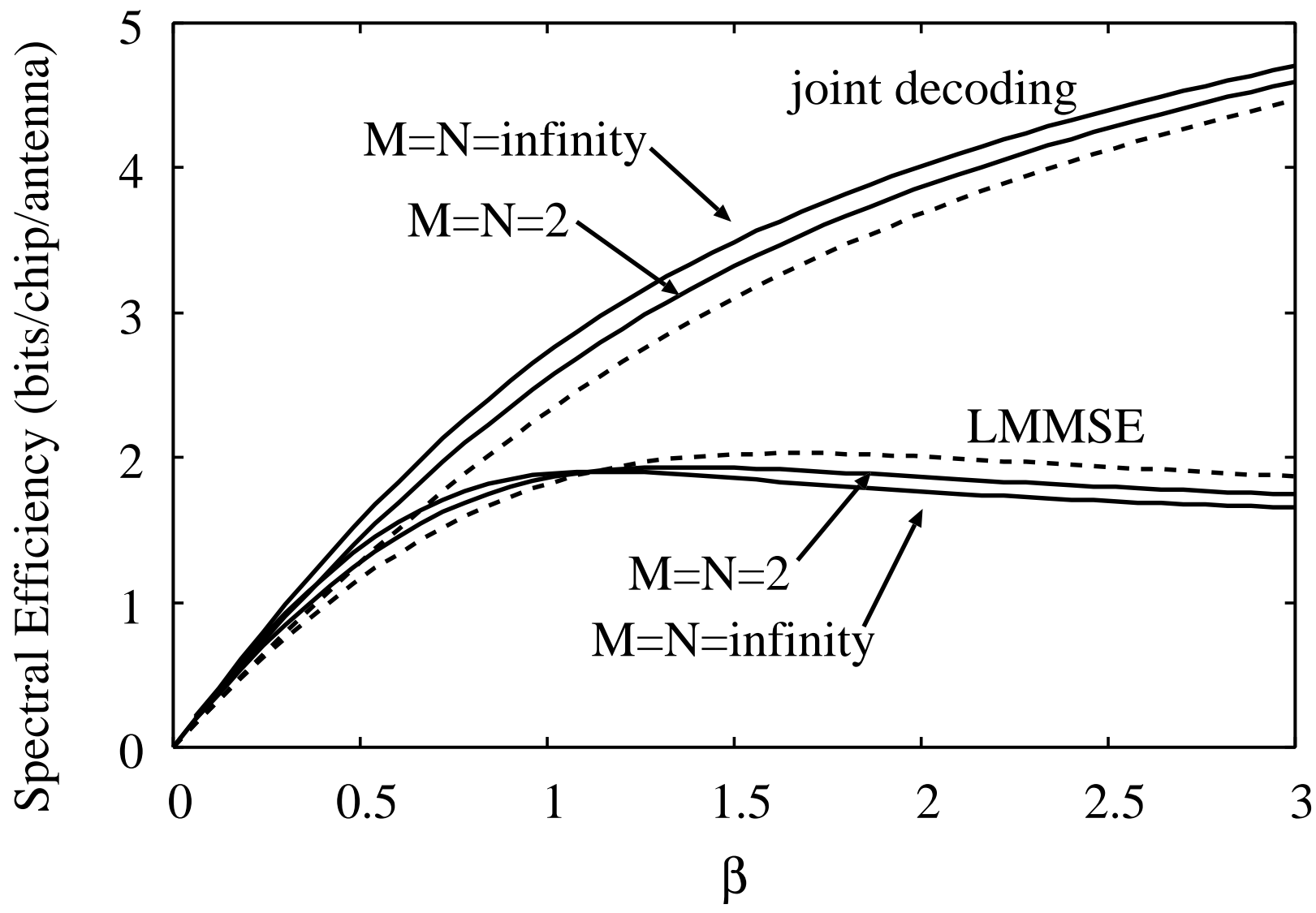

Fig. 3. Spectral efficiency per transmit antenna of the MIMO DS-CDMA channel versus $\beta$ in the case of the Gaussian data modulation. The solid lines are the spectral efficiencies per transmit antenna in the case of the STS scheme. The dashed lines represent the spectral efficiencies per transmit antenna in the case of the TS scheme. The dashed lines overlap for $M=N=$ $2, \ldots, \infty . P / N_{0}=10 \mathrm{~dB}$. 


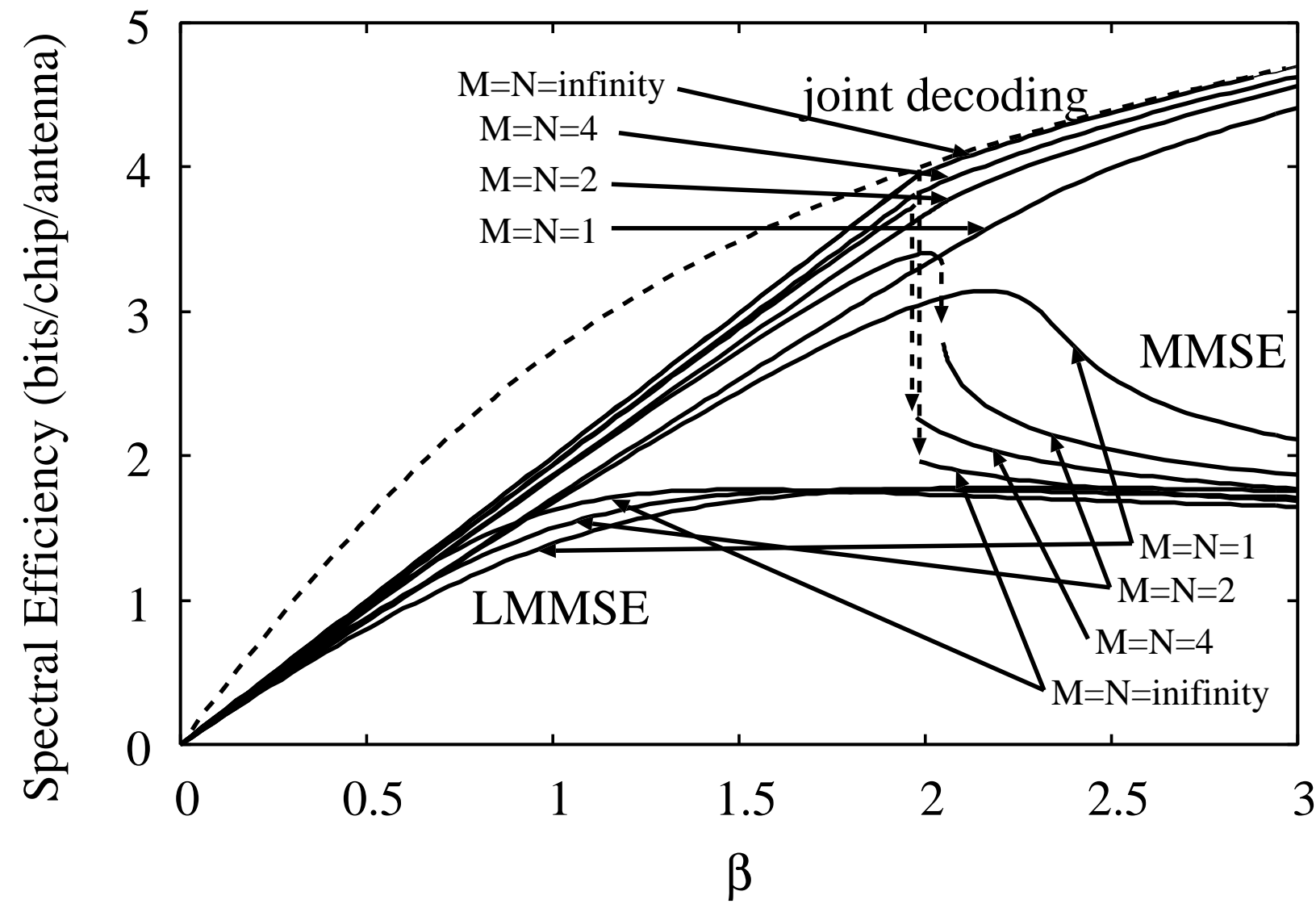

Fig. 4. Spectral efficiency per transmit antenna of the MIMO DS-CDMA channel with the QPSK data modulation scheme versus $\beta$ in the case of the STS scheme. The dashed line is the maximum spectral efficiency per transmit antenna of the MIMO DS-CDMA channel with the Gaussian data modulation scheme and the STS scheme for $M=N=\infty . P / N_{0}=10 \mathrm{~dB}$. 